\title{
Small Scale Rollout of PV Systems in Chikwawa District, Malawi: Remote Monitoring System Effectiveness
}

\author{
Million Mafuta, ${ }^{1}$ Peter Dauenhauer, ${ }^{2}$ and Timothy Chadza ${ }^{1}$ \\ ${ }^{1}$ Electrical Engineering Department, University of Malawi, The Polytechnic, Blantyre, Malawi \\ ${ }^{2}$ Electronic and Electrical Engineering Department, University of Strathclyde, Glasgow, UK \\ Correspondence should be addressed to Million Mafuta; mmafuta@poly.ac.mw
}

Received 19 October 2016; Accepted 22 February 2017; Published 19 March 2017

Academic Editor: Sundaram Senthilarasu

Copyright (C) 2017 Million Mafuta et al. This is an open access article distributed under the Creative Commons Attribution License, which permits unrestricted use, distribution, and reproduction in any medium, provided the original work is properly cited.

\begin{abstract}
Off-grid solar photovoltaic systems in Malawi are deployed increasingly as the primary option for rural public infrastructure such as primary schools and health centres. Overall, grid-connected electricity access has remained stagnant at around $9 \%$ with only $1 \%$ of rural population connected. To improve the technical sustainability of such systems, a novel remote monitoring technology utilising Wireless Sensor Networks was installed and the systems were monitored over roughly one year. This paper has described the technical design, performance, and benefits received from deployment of the technology. Furthermore, it has evaluated the cost implications for a larger scale rollout and potential benefits.
\end{abstract}

We dedicate this work to Mr. McEnroe Mulera who was a great asset to the project and will be missed

\section{Introduction}

Modern energy access is a critical input for economic development. It was widely regarded as a key enabler to many social development outcomes even before its inclusion as part of the seventh Sustainable Development Goal in 2015 [1]. The provision of the first kilowatt of electricity can be transformational for a community, household, or individual which in most cases depends on biomass or fossil fuel energy source.

Focusing on electricity access alone, nearly 1.2 billion people lack access to electricity primarily in lower income countries around the world like in South East Asia and SubSaharan Africa [2]. In these regions, off- and mini-grids are expected to provide up to $70 \%$ of the new electricity access in the rural areas [3]. There is a great interest internationally to introduce private market investment and discipline to solve this problem. Tens of billions of dollars is expected to be required to accomplish universal access. Meanwhile, many off-grid projects continue to suffer from sustainability challenges, lowering the potential returns and increasing the risk investments. For example, in a recent review of 23 small scale projects, conducted at the 12-24-month mark, it was found that only $48 \%$ were fully functional [4]. This echoed by the experience from the World Bank, where, compared to grid extension schemes, off-grid programmes were less successful due to higher costs and lower benefits. Schemes have suffered from technical shortfalls and the economic rationale of pursuing off-grid versus grid extension was questioned [5].

Potential solutions are inherently multidisciplinary. With knowledge of the microeconomics of the individual markets where projects are deployed, the need for skills and a capable organization, and the level of coordination of the national and local governance, can all impact the success of off-grid projects. In higher-income countries, technologies such as Energy Management Systems (EMS) involving Supervisory Control And Data Acquisition (SCADA) have been deployed since the 1960s and can therefore be considered mature [6]. For a variety of reasons, such as cost and design for higher voltage grid infrastructure, these technologies are not immediately applicable to an off-grid developing country context. The rise of smartphones, cloud computing, and relatively robust networks in developing countries [7] has created a new opportunity for off-grid system monitoring. This paper 


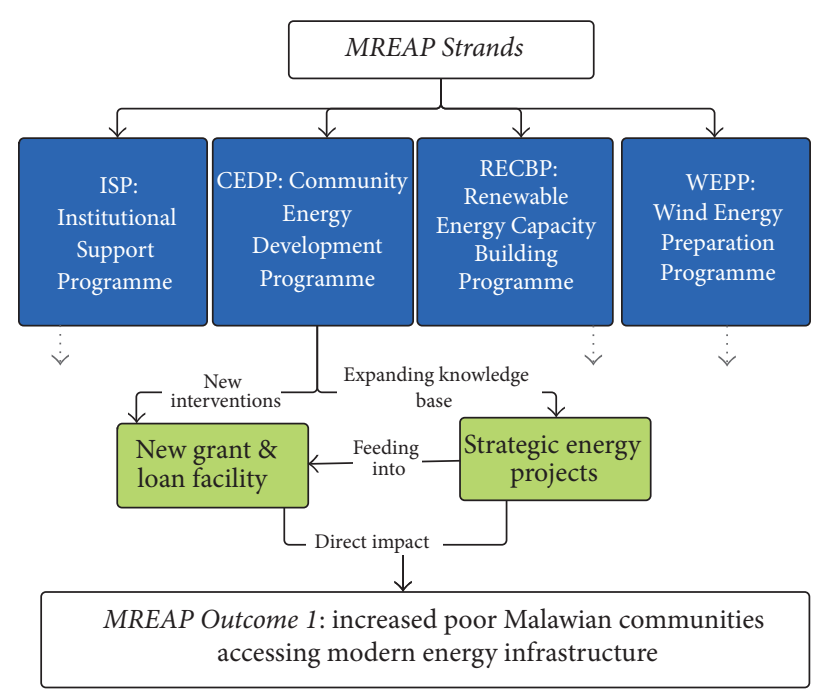

FIGURE 1: MREAP organization and SEPs.

demonstrates the use of a remote monitoring system in an off-grid context in Malawi and evaluates the benefit such operational data can provide towards sustainability of these systems.

An alternative to adoption of existing technologies is the investigation of new techniques to monitor and ultimately provide smart control of off-grid systems. This will have the potential to greatly improve the sustainability outcomes of these systems. Without new research, prototyping, and testing of new technologies, the available solutions are reduced. Given the rapid recent development of computer and communications systems, there is value in pursuing whether they can provide a significant impact-both directly in terms of sustainability improvements and generally in terms of their ability to generate firm evidence on how these systems are used.

This paper makes several novel contributions to the literature. Firstly, it thoroughly details the deployed Wireless Sensor Network (WSN) system and analyses the performance of the technology in an off-grid energy access scenario. With little available literature in this context, the results are a valuable case study for future similar deployments. Secondly, it presents and analyses the output of the WSN system and power system performance data for ten off-grid solar PV systems in Malawi. Since most of past off-grid PV systems lacked the sensing capability, available datasets are extremely rare. The analysis of operational data reveals daily and weekly trends as well as faulted situations to which practitioners rarely get access. Finally, these results are utilised to build an argument for the sustainability and related benefits derived from the use of the technology. The paper views this as a critical perspective which all stakeholders from policymakers to practitioners need to consider when planning offgrid solar PV system deployments.

1.1. MREAP's Strategic Energy Project in Solar PV. The Malawi Renewable Energy Acceleration Programme (MREAP) was funded by the Scottish Government to explore multiple yet coordinated strands of work to improve prospects for renewable energy in Malawi [8]. The programme ran from 2012 to 2015 and had two key outcomes: improving the enabling environment and improving access rates to affordable and appropriate energy for poor Malawians [9]. Much of the emphasis of MREAP has been on demonstrating and improving the model for community energy in Malawi, especially in rural areas where electricity access rates are only $5 \%[2]$.

MREAP had four overall work streams based on needs identified for the sector [10]: Institutional Support, Renewable Energy Capacity Building, Wind Energy Preparation, and Community Energy Development. The latter work stream was organized into two complementary activities. Firstly, four strategic energy partners piloted four separate technologies (off-grid solar photovoltaic (PV), micro-hydromini-grids, Biogas Digesters, and improved cook stoves). Each strategic energy partner had extensive past experience in deployment of these technologies in which the MREAP would provide further opportunity to learn what worked most effectively. Secondly, a facility to provide funding to new projects would be established. This would build upon learning from the Strategic Energy Projects (SEPs). The arrangement is summarized in Figure 1.

During the system deployment, the primary objective of the Centre for Water, Sanitation, Health, and Appropriate Technology Development (WASHTED) at the Polytechnic in Blantyre, as a Strategic Energy Project lead for off-grid Solar PV, was to improve on the sustainability model in Malawi for community managed off-grid solar PV. Overall, four locations in Chikwawa district were selected through needs assessment process and projects installed through 2014 and early 2015 [11, 12]. The design included deployment of a unique remote monitoring (RM) solution as a measure to bolster technical sustainability of the project.

1.2. Operation of Rural Solar PV Systems and Sustainability Challenges. Sustainability can be defined as "the perceived 


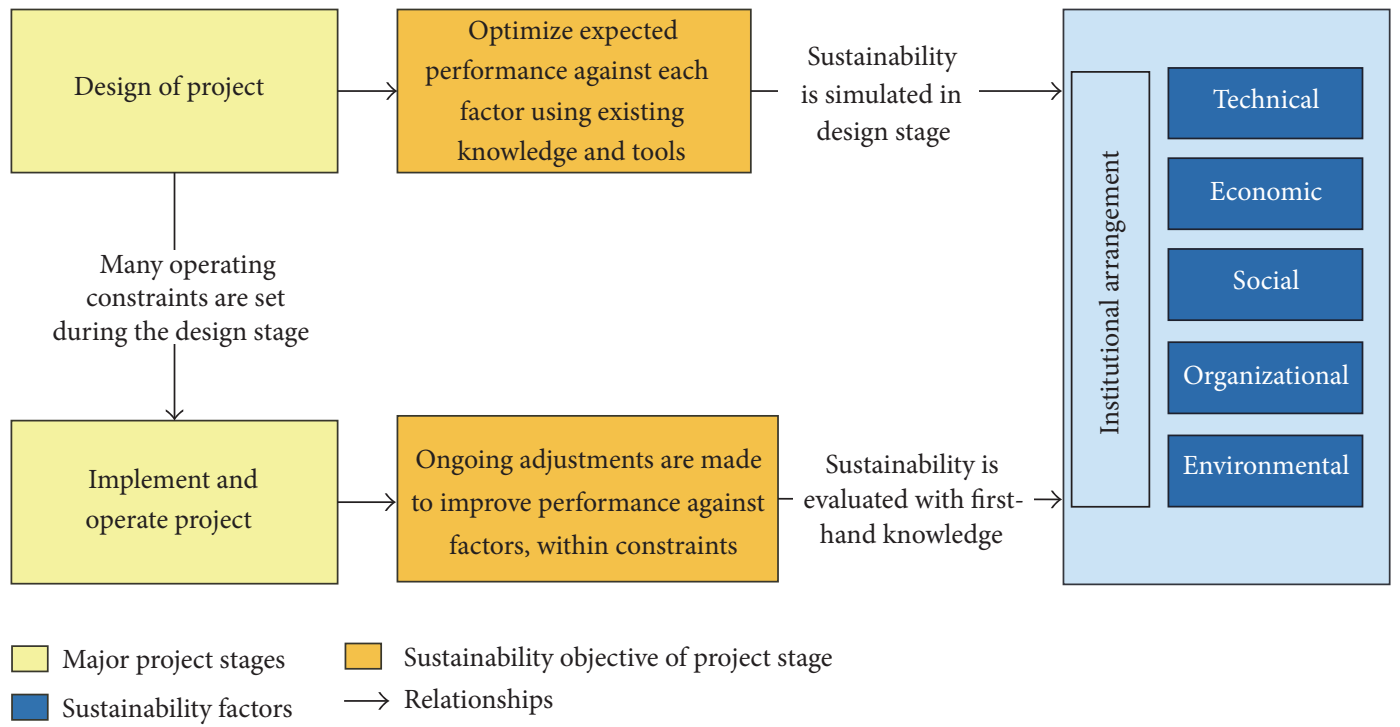

FIGURE 2: General sustainability framework.

potential for a system or project to endure, build a selfperpetuating capacity within a community, and ultimately reach the end of its predefined lifespan or evolve into another beneficial form" [13]. Various iterations on models and general frameworks for sustainability have been developed. For example, some are based on hands-on experiences with Renewable Energy Technology (RET) deployment [13, 14] or adjusted for use from top-down indicator frameworks $[15,16]$. Finally, a class of guidance tools on project design or operational challenges exists which highlights the various forms of sustainability issues off-grid projects face [17].

Another angle that adds complexity is the externalto-project environment, which the frameworks all discuss to some degree. Besides countering sustainability issues in identified domains at the project-level, technical, economic, social, organizational, and environmental, the project will be subject to an institutional environment both local and national (even international) which may help or hinder the project as the case may be. This may be described as "institutional" sustainability and may include, for example, legal and regulatory enablers or hindrances. A sustainable project implementation process will recognise the sustainability link between the design and operation stages, where specific design has a significant impact on future operations. Figure 2 shows the general sustainability relationship.

Historically, project-level sustainability of off-grid solar PV projects has been an ongoing challenge in Malawi. In 2012, there was an estimated 7,000 PV systems present in the country, though many are known by practitioners not to be fully functional [18]. The MREAP steering group concluded in 2012, at the onset of the programme, that not only was very little information known about the existence and performance of these projects, but also those that were known had challenges in sustainability. The evaluation's sustainability analysis considered the following factors enabling environment: financing, technical viability, and capacity. A host of issues raised through the report included weak enabling environment (undefined or low responsibility for maintenance and operations of equipment), weak quality standards, warranties, contracts and insurance, low financing opportunities for energy services, insufficient project financial performance, and lack of RET standards [19].

For solar PV systems in Malawi, no data are currently known to be widely available on the technical system operation and performance. Analysis of such data would shed light on how these systems are used or, as the case may be, misused. Internationally, a widely cited sustainability study in 2001 by Nieuwenhout et al. [20] had mixed results on technical sustainability, with $42 \%$ of systems studied not fully functional. Comprehensive cross-country studies of sustainability of off-grid systems are in short supply, and recent reports continue to cite failures and barriers to scaling $[21,22]$.

Recently, a solar PV sustainability study carried out in 2014 found that for lighting loads, only $42 \%$ of 188 rooms surveyed throughout Malawian public service institutions were meeting usage expectations [23]. This implies two opportunities for RM technology which are addressed in this paper: firstly, to better understand how off-grid PV systems themselves operate in Malawi and, secondly, to use RM technology to better improve sustainability of these systems.

1.3. Remote Monitoring Technology Overview. Unique to the context of off-grid power system in developing countries, the technology employed Wireless Sensor Network (WSN). This system generated detailed data from several nearby, but asynchronous, systems and transmitted this using a Global System for Mobile (GSM) communication network to a central monitoring point. WSN is flexible at both software and hardware levels. The hardware level permits integration of many different sensors and actuators, while the software can easily be modified to suit the user specifications. Moreover, it is a low-cost and low-power consumption technology. These features have accelerated the application of WSNs in many 


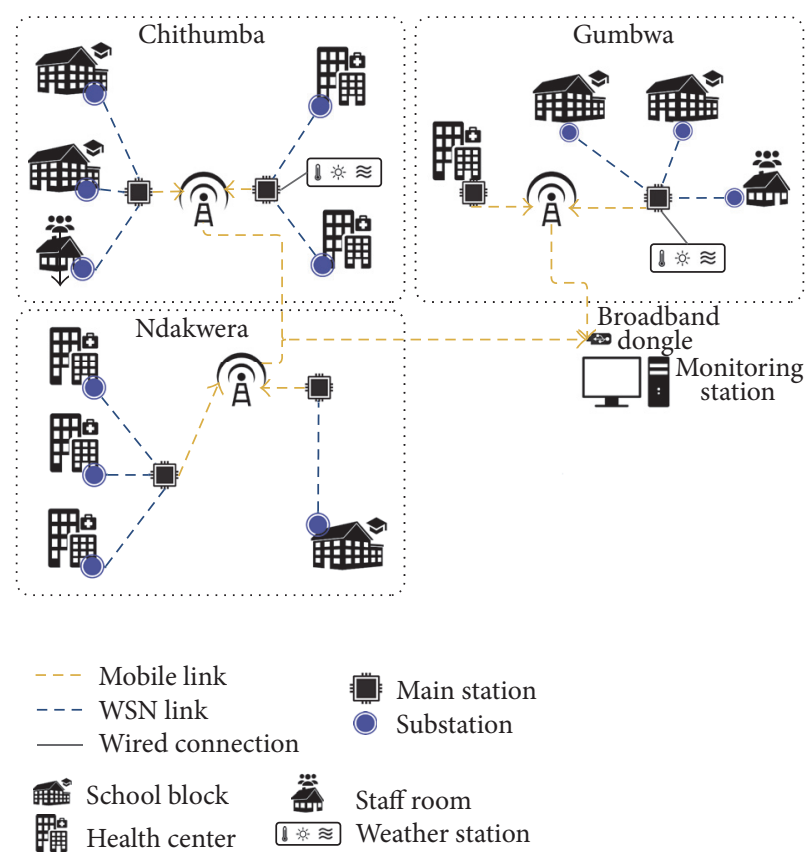

FIgURE 3: A conceptual model for the RM system.

areas including precision agriculture [24-26], wildfire detection and reporting [27-29], and PV condition monitoring and reporting [30-32]. The reviewed literature is focused primarily on analysing performance and demonstrates the effectiveness of the WSN technology. The applications are usually located in rural and remote areas where physical access is both challenging and costly. However, in the literature there exists little discussion around the impact it has on the actual applications. This paper seeks to provide some of this analysis in the area of off-grid power applications.

The paper is structured as follows: Section 1 discusses the background to the study, context of energy access, and sustainability of off-grid projects and includes a short WSN technology literature review. Background to the Malawi Renewable Energy Acceleration Programme (MREAP) is provided to better understand the context of the approach taken. Section 2 discusses the remote monitoring (RM) system, which includes the WSN subsystems, in extensive detail. Sections 3 and 4 form the body of results starting with a description of the dataset and leading into the presentation and analysis of this data. Section 5 builds an argument for the cost and benefit of RM towards improving sustainability of the projects. Section 6 draws the conclusion from the discussions.

\section{Overall RM System Design}

As depicted by Figure 3, the RM system was divided into two main elements: Remote Stations (RSs) in Chikwawa District which comprised Chithumba, Gumbwa, Ndakwera, and Dolo (not shown here) and the Central Monitoring Station (CMS) at the Polytechnic in Blantyre. These two elements were interlinked by a cellular network. Each RS had several nodes that captured real-time performance parameters at 15-minute intervals. The parameters included PV array voltage, battery bank voltage, charging current, and load current. Readings were sent from the CMS through Short Message Service (SMS).

In order to reduce the SMS charges, the design opted to assign one independent node to collect the aggregated parameters from the particular RS and forward them to the CMS. The CMS itself aimed at receiving the information from the RS, storing it into a database, and then displaying the performance data onto a computer screen. The CMS was also capable of alerting the management personnel through an SMS when the PV system performance parameters swayed away from the normal operating points.

2.1. Remote Station (RS) Design. The RS mainly consisted of the PV installation and the RM equipment based on WSN. Waspmote node by Libelium [33] was chosen since it is an open source model offering an advantage of low cost when compared to the proprietary solutions. The main feature of the Waspmote node is the availability of XBee transceivers which provides the flexibility in aggregating data from polydisperse locations within the same infrastructure establishment. The Waspmote board has the following other characteristics: first, low-power consumption of $70 \mathrm{nA}$ when in hibernation; second, architecture flexibility that permits additional sensors to be integrated easily; third, the provision of General Packet Radio Service (GPRS) module and Secure Digital (SD) card slots; and fourth, the availability of a RealTime Clock (RTC) [33]. All the four RSs had similar design and implementation, so for simplicity the analysis focuses on the Gumbwa system. There were two main stations at the Gumbwa site communicating with the CMS located at 


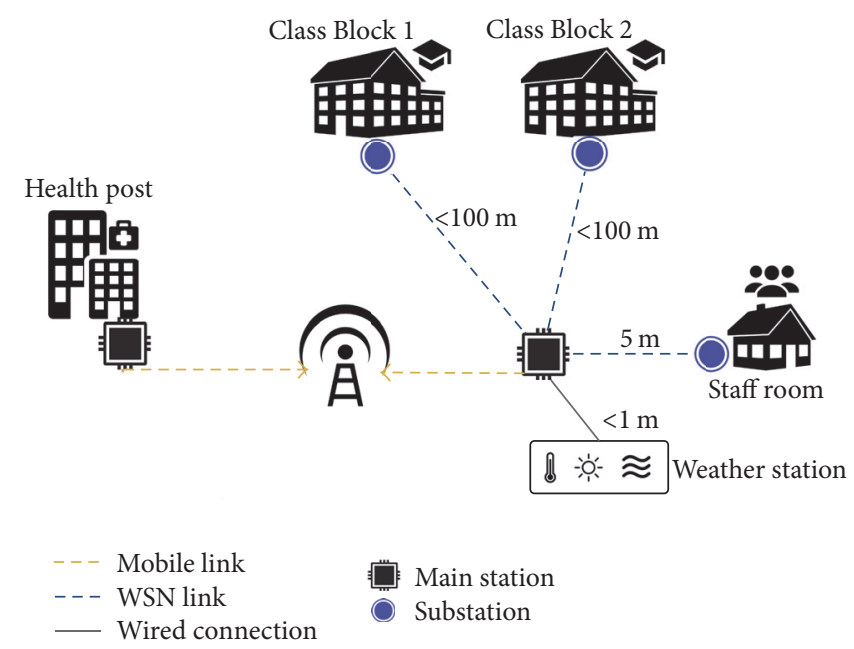

FIgURE 4: Architecture of the Gumbwa Remote Station.

the Polytechnic in Blantyre (46 km away) via the cellular network as depicted in Figure 4. One of the two main stations had four substations, three of which measured and relayed the PV performance parameters to the main station. The other substation measured and communicated the weather parameters to the main station.

This local communication (substation node to the main station) was possible using a ZigBee transceiver module which is based on the Media Access Control (MAC) and Physical (PHY) layers of the IEEE 802.15.4 standard. The transceiver operates in the unlicensed Industrial, Scientific, and Medical (ISM) radio bands which are $868 \mathrm{MHz}$, $915 \mathrm{MHz}$, and $2.4 \mathrm{GHz}$ depending on the region $(2.4 \mathrm{GHz}$ for the case of Malawi). The main focus of ZigBee protocol is on low cost and low-power consumption. Nevertheless, the low-power consumption feature of the ZigBee protocol imposes the limitation on its coverage range which is in the order of $60-100 \mathrm{~m}$ when Line-Of-Sight (LOS) mode of communication is used [34]. As shown in Figure 4, this limitation resulted in the deployment of two independent main stations at the Gumbwa site since one of the four PV installations was about $139 \mathrm{~m}$ away from the rest. Moreover, it was impossible to achieve a direct LOS communication since such deployments were mostly indoor.

As depicted by the flowcharts shown in Figures 5 and 6, each substation was configured to capture four sensor data points at intervals of 15 minutes. These were aggregated and sent to the main station every hour. Upon receiving two such datasets from each substation (translating to a two-hour interval), the main station aggregated and prepared them to suit the SMS transmission system. The data were then sent to the CMS as an SMS. In order to send an SMS, the main station was equipped with a GPRS module that consumes up to $2 \mathrm{~W}$ of power when active [33]. In view of this high power consumption problem, it was judicious to switch on this module only when sending an SMS. This was handled at the software level of the system. It was observed that using this power saving strategy, coupled with sending an SMS only after a time lapse of two hours, the RM system could operate seamlessly for over two months without recharging a dedicated 6.6 Ah lithium battery. Nonetheless, in order to improve the robustness of the RM system further, every Waspmote node was equipped with a $2.5 \mathrm{~W}$ solar panel independent of the main PV installation system. The panel was connected through a dedicated socket available on the Waspmote board.

2.2. Central Monitoring Station (CMS). The CMS is the core element of the remote monitoring system. It was used to aggregate and process data relayed to it by all the RSs deployed in rural areas of the Chikwawa district.

The CMS runs from a personal computer equipped with a Linux operating system. Linux was chosen because, as an open source software, it can be a solution to a low-cost RM system suitable for a low resource setting in developing countries. Figure 7 outlines a conceptual model of the CMS. It shows how data received by the Broadband dongle was processed and graphically evaluated. A more detailed description of the same is depicted by the flow chart in Figure 8.

The data from the RS were captured directly by the Broadband dongle contained in the CMS. Due to the limited storage capabilities of the Subscriber Identity Module (SIM) card inserted into the Broadband dongle, a FrontlineSMS interface was used to push the data from the dongle to a MySQL database. This interface was chosen as a gateway between the dongle and the database because it is a free open source software and provides a more user-friendly Java FrontlineSMS back-end based browser [24].

In order to maximize the usage of the SMS transmission channel, the RS concatenated the data received from all substations into a single string. Consequently, the raw data were stored in the database by the SMS gateway in the main table. Since this data was not in the appropriate format and syntax, a Hypertext Preprocessor (PHP) script was developed to process the data into specific tables for easier viewing. This script was automated to refresh every 5 minutes so as to process any incoming data within such period. As a management system, it was essential to analyse the PV 


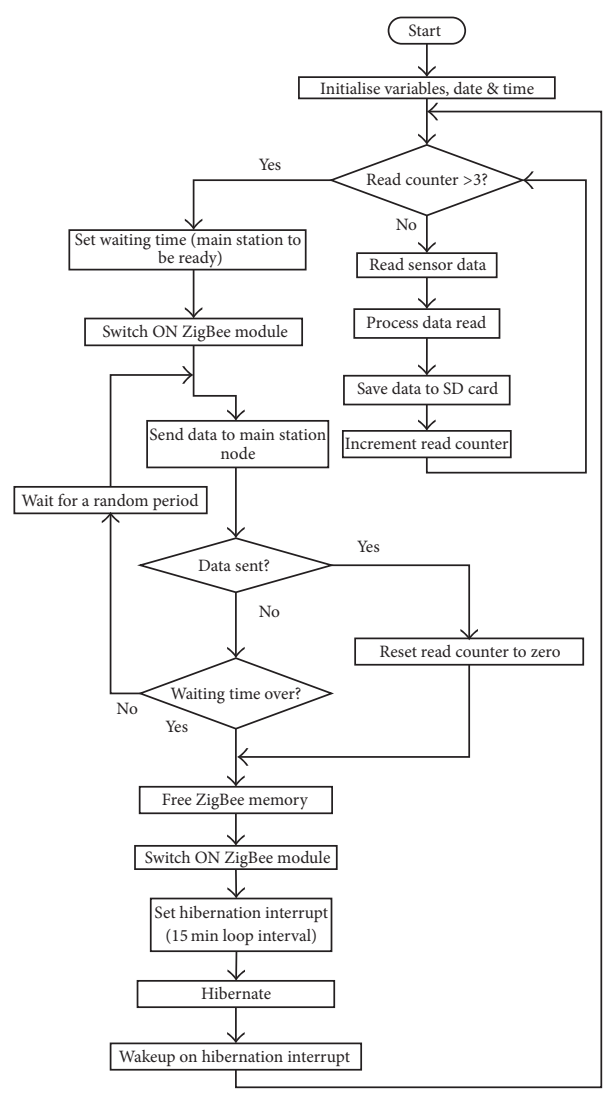

Figure 5: Flowchart for substation node.

system performance metrics graphically on a main page. This required plotting of the data using graphing library that can be embedded within or uses PHP. JpGraph is an ObjectOriented Graphing library for PHP versions 5.1 to 5.5 and was used for real-time plotting.

It is worth noting that using this graphing tool it was possible to view both current and historical PV performance parameters. For instance, the monitoring personnel were able to view data within the customised time periods. Whenever that was not required, the default or preset periods were chosen to be "last 24 hours," "last 7 days," "last 30 days," and "last 730 days."

Additionally, the CMS, housed at the Polytechnic, permitted the monitoring personnel to receive alert messages for the RSs wherever they were through their mobile phones. A PHP script was implemented in the CMS to check any abnormal conditions of RSs. Whenever abnormal trends were noted by the CMS system, the personnel were alerted for immediate remedial action, either by visiting the remote site or by calling local users.

2.3. Remote Monitoring (RM) System Costs. The main costs for the deployed RM equipment over the lifetime of the project comprised both capital and operating expenses. Operating costs were mainly due to SMS charges. These ranged from $\$ 276$ to $\$ 317$ (all costs originally recorded in British Pound Sterling and converted to US Dollars using the December 1, 2014, exchange rate (GBP $0.6379=$ USD 1.00)) per site depending on the number of main stations per site, SMS sending rate, and the mobile network used. Each of the three sites had two main stations while one (Ndakwera) had only one main station. Typically, an SMS would be sent from main station to CMS every two hours.

An additional operation cost was incurred through monitoring and maintenance activities. At some locations there was no maintenance cost. At others it was as much as $\$ 705$ per year when more site visits were needed. Gumbwa required three site visits per year, the most frequent one of all the locations. The advantage was that site visits were prompted by the observed conditional data since the RM was able to report its own health status. The yearly SMS and maintenance costs for all four locations were $\$ 3,700$ or roughly $\$ 925$ per location.

Capital costs, not inclusive of solar PV system costs, were scaled to the number of independent PV stations required at the site as well as the distance from each RS unit. This included the combination of microcontroller boards, Current Sensors, Voltage Sensors, GPRS modules, dedicated rechargeable batteries, SD cards, dedicated mini PV panels, and enclosures. For reference, installed PV capacity at these sites varied between three and five asynchronous solar PV systems with a total capacity between $0.85 \mathrm{kWp}$ and $2.1 \mathrm{kWp}$. Each location required at least one RS main station costing either $\$ 337$ or $\$ 608$ depending on if it required on-board sensing. This was the case in the smaller installation where there was only a single PV system. Where multiple PV installations monitored were within proximity of the WSN, 


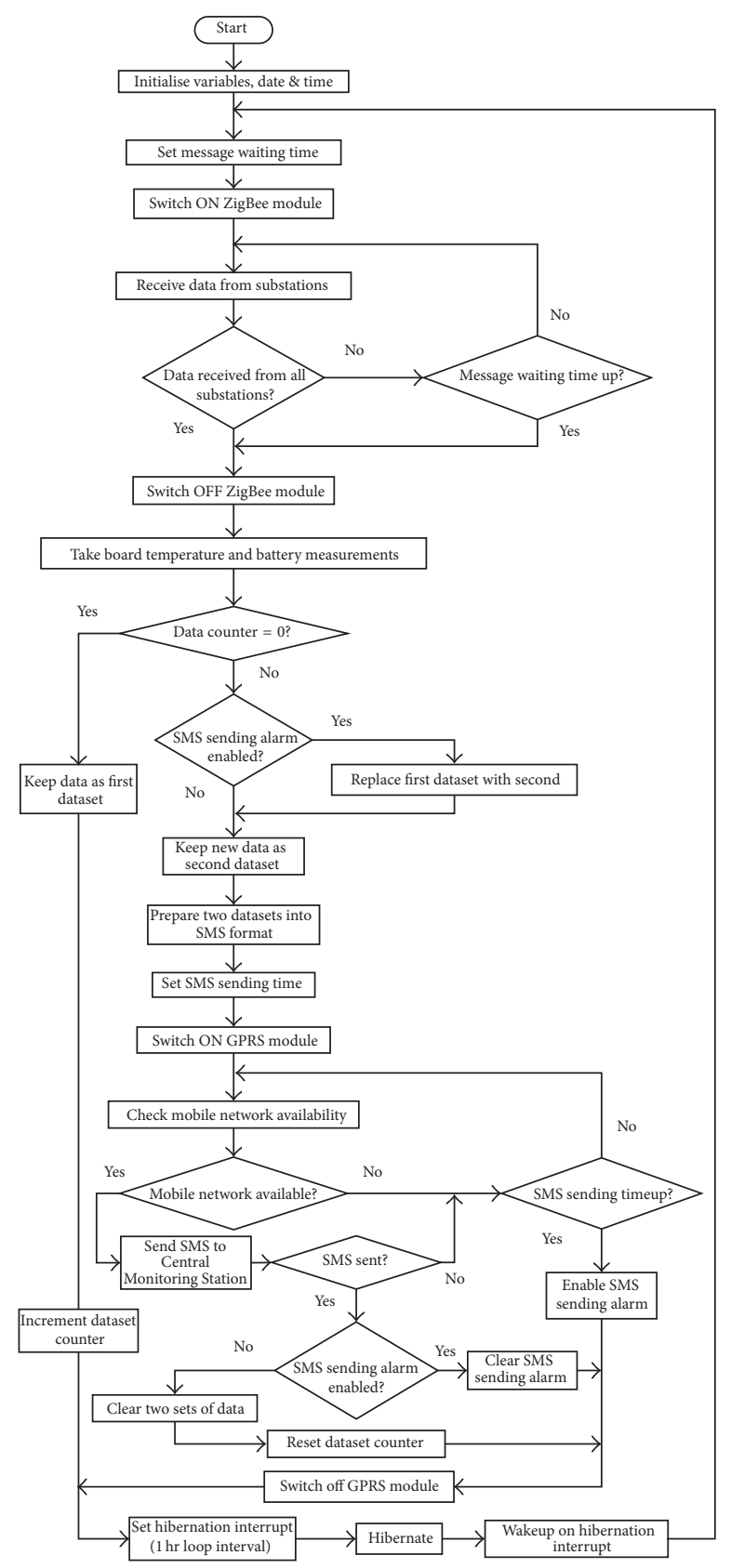

FIGURE 6: Flowchart for main station node.

additional RS-substations were connected at $\$ 361$. Figure 9 shows the RM deployment configuration for both single and multi-PV systems. The capital cost of the largest RM system with six RS-substations was $\$ 2500$. In total 5x multistation RS-Main, 3x single-station RS-Main, 13x RS-substations, and $1 \mathrm{x}$ automatic weather station were installed. Installation costs for each of the subcomponents was $\$ 140$ on average.

For research purposes, an automatic weather station was installed at Gumbwa in order to investigate the effect of weather parameters on PV generation capability. Additional equipment required included sensor mounts, solar irradiance sensor, humidity sensor, anemometer, wind vane, pluviometer, temperature sensor, and atmospheric pressure sensor. The total cost for this station was $\$ 994$.

Finally, the cost for the CMS was $\$ 2,344$. This constituted an Uninterruptible Power Supply (UPS) unit, Broadband dongle, and a personal computer (500 GB HDD, 2.6 GHz dual core processor, and 4 GB RAM).

2.4. Description of the Installation. The proper integration of the RM system to the PV installation system was tantamount to its operation and the correct reporting of the PV 


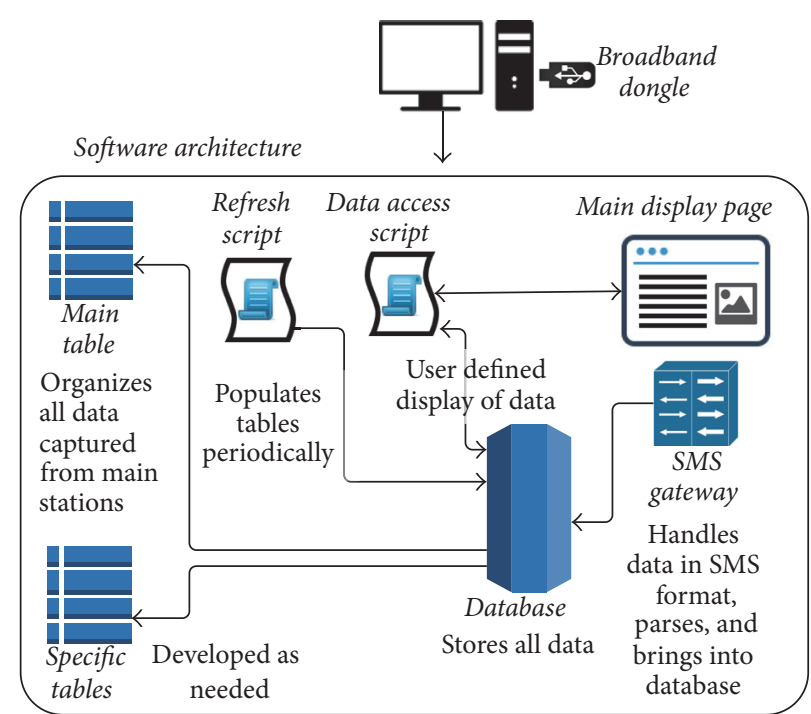

FIgURE 7: Conceptual model for the Central Monitoring Station.

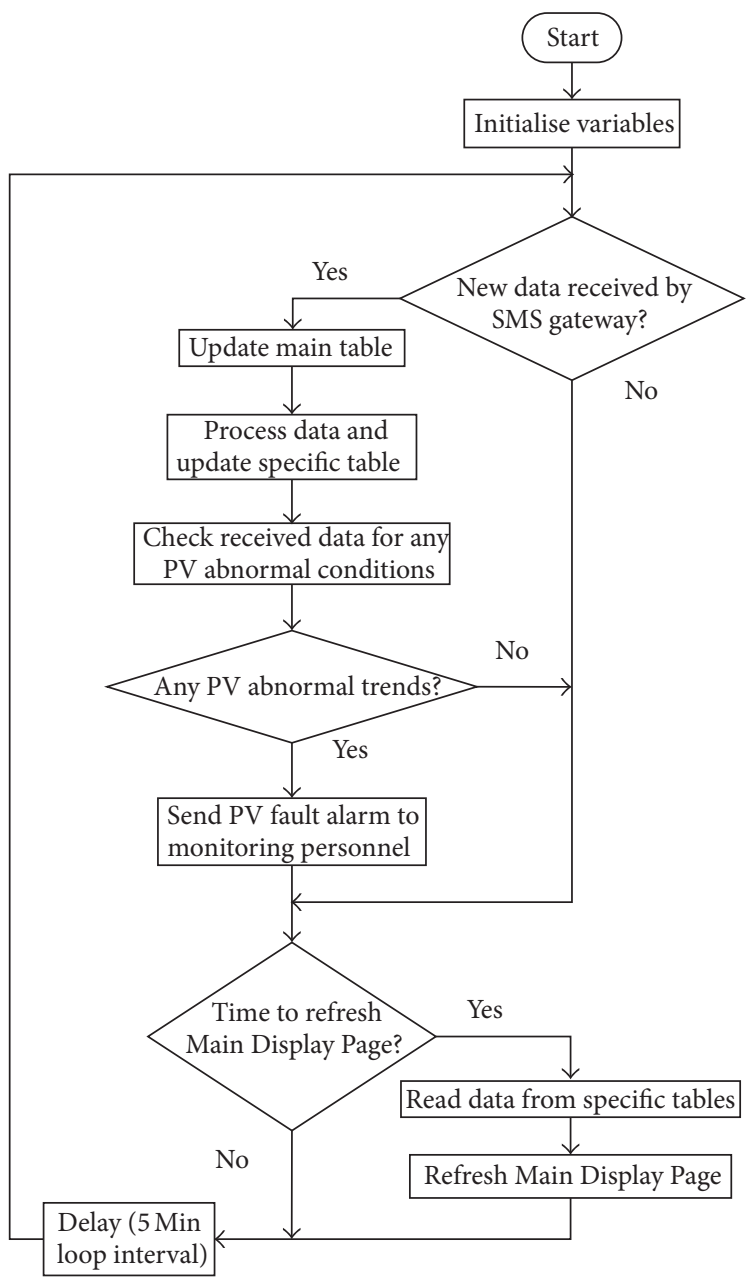

FIgURE 8: Flow chart for the Central Monitoring Station. 


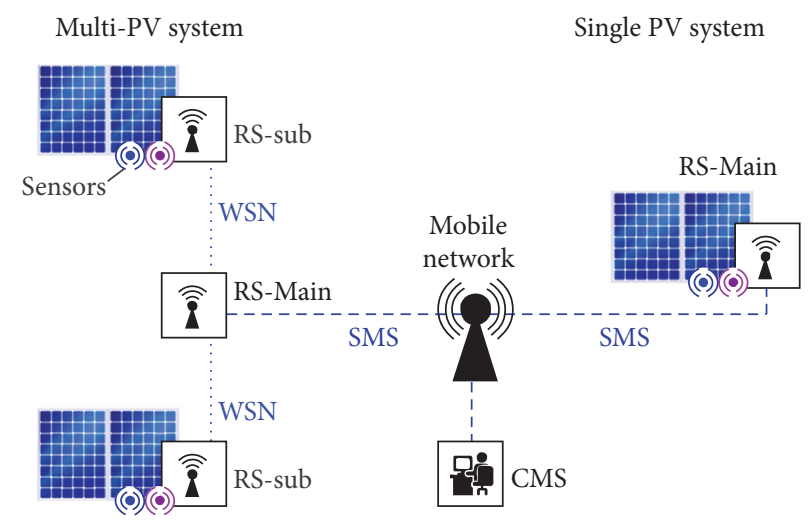

FIGURE 9: RM deployment single PV systems versus multi-PV systems.

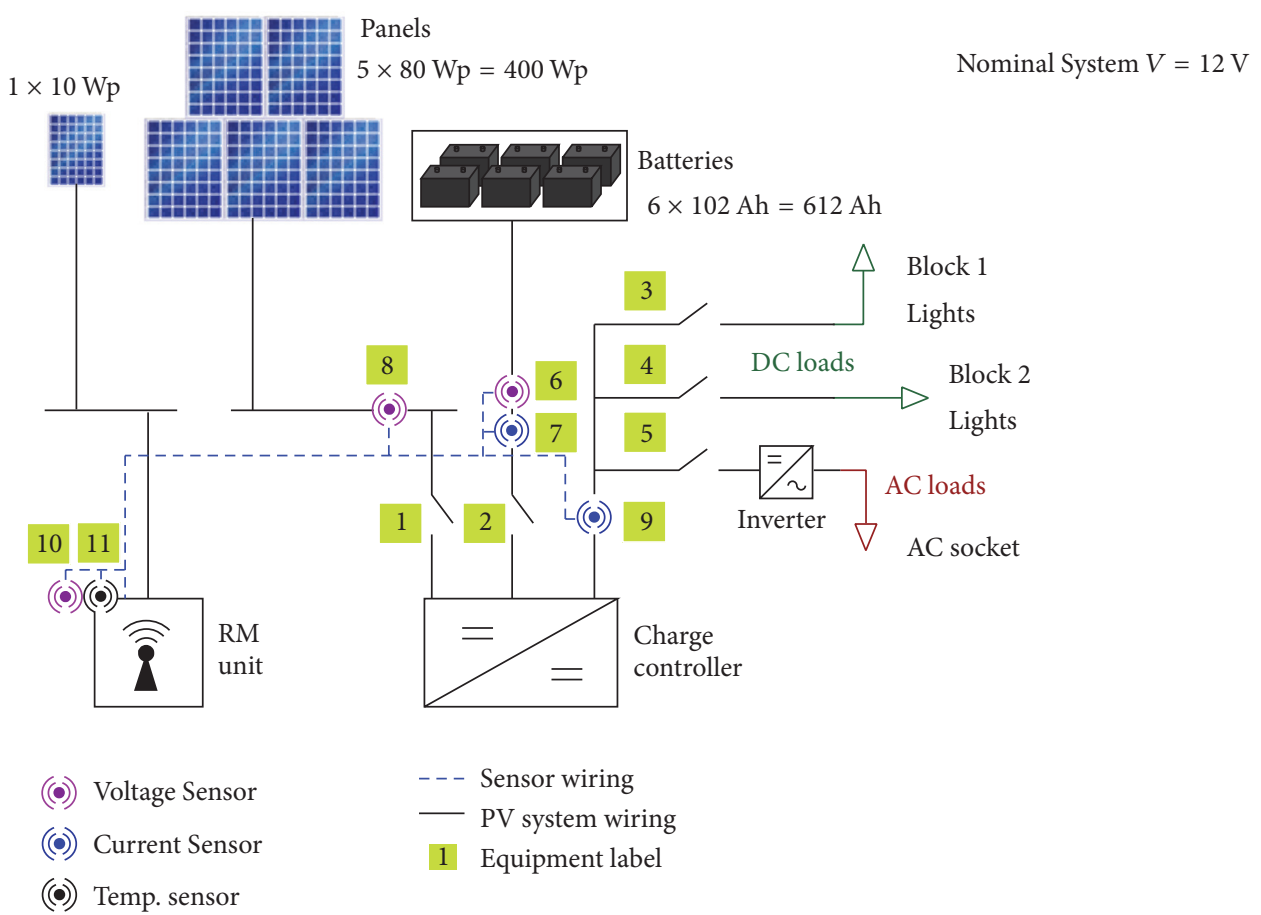

FIgURE 10: Gumbwa classroom 2 PV and RM system diagram.

performance parameters. In view of this fact, the design for both RM and PV systems was a coordinated task between the RM and the PV specifications and requirements. For instance, all the RSs employed the 1122 Current Sensor and the 1135 Voltage Sensor both by PHIDGETS Inc. [35] due to their low cost as compared to other commercially available sensors with the same or similar capabilities. According to [35], the 1122 Current Sensor measures Alternating Current (AC) up to 30 Amps and Direct Current (DC) level of up to +/-30 Amps whereas the 1135 Voltage Sensor accurately measures a differential voltage level of up to $+/-30 \mathrm{~V}$. It was therefore important to understand these limits when designing the PV systems. However, since the core business of the project was to do with the PV system rather than the RM system, a concerted effort was exercised not to compromise the PV system configuration at the expense of the RM system.

Figure 10 shows, as an example, how one PV installation node at Gumbwa site was configured alongside the RM system. Based on the anticipated load demand, the system required five $\mathrm{PV}$ panels of $80 \mathrm{Wp}$ each. With each panel having a short circuit current of $5 \mathrm{Amps}$, this meant that the maximum total current delivered to the system was 25 Amps. An assumption of a 30\% factor of safety yields a charge controller size of 32.5 Amps which unfortunately was not available on the market, resulting in a 40 Amp charge controller being opted for. Nevertheless, a 32 Amp Circuit Breaker (CB) 1 was connected between the PV array and the charge controller in order to protect the panels in case of a 


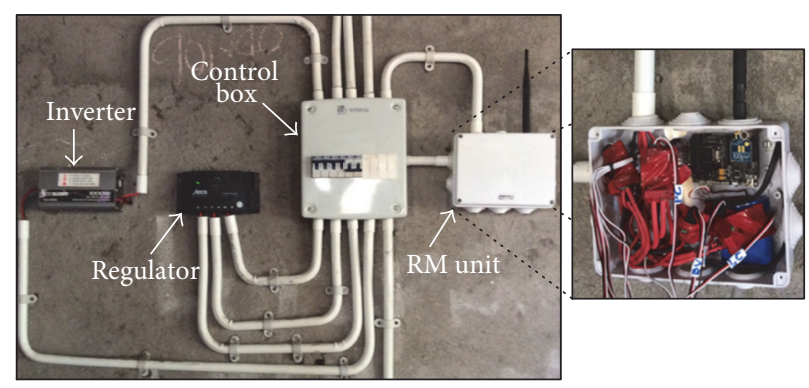

FIgURE 11: Completed installation of a Remote Station.

prolonged short circuit fault at the controller port. In order to continuously check the health status of the PV array it was important to install Voltage Sensor 8 just before the CB 1.

Furthermore, a battery bank comprising six batteries, $102 \mathrm{Ah}$ each, was required at this Gumbwa node. Voltage Sensor 6, as shown in Figure 10, was then installed to monitor the level of the voltage for the battery bank. On the other hand, Current Sensor 7 was used to measure the unidirectional charging current from the charge controller to the battery bank. Again it was important to isolate the battery bank from any possible short circuit in the control box by installing a 32 Amp CB 2.

Finally, the charge controller was required to supply the three sections of the load. As shown in Figure 10, these load sections were (1) lights to classroom 1, (2) lights to classroom 2, and (3) AC sockets for television usage. Each of these sections demanded roughly 9 Amps, 7 Amps, and 9 Amps, respectively. Therefore, under normal operation of the system, the total load current was not supposed to exceed 25 Amps. This permitted the use of Current Sensor 9, whose recommended upper current limit was $30 \mathrm{Amps}$ [35], to measure the total load current. It was also important to isolate the entire PV system from the possible short circuits at the load side by incorporating CBs 3, 4, and 5, all rated 16 Amps.

Figure 11 shows a picture of a complete installation of one RS. As shown in this figure, the RM unit was weatherproofed in an IP65 rated polyvinyl chloride (PVC) box. The right hand side of this figure shows an opened RM unit box housing a microcontroller board, Voltage Sensors, and Current Sensors. All sensors were wrapped in an insulation tape to avoid possibilities of short circuits within the box.

\section{Operation: System Performance}

Data were captured for ten systems over three projects in Chikwawa district, Malawi. Projects are identifiable by location: Gumbwa, Ndakwera, and Chithumba. Each project has both a primary school and a health facility in close proximity which enables the WSN based RM equipment to perform as designed.

At Gumbwa, data from two classroom blocks have been captured along with a staff room and a nearby health post. At Ndakwera, one classroom block was monitored. Likewise, the health centre nearby includes Outpatient Department (OPD), recovery ward, and a maternity ward which are monitored. At Chithumba the maternity ward and OPD at the health centre are monitored. Overall, 166,927 observations were recorded on 10 separate subsystems. The RM system was up for 62,460 hours of the 67,673 hours recorded. This translates into an estimated reliability of $92 \%$ (calculated as hours online/hours total). The weather station is located at Gumbwa.

The utilised measurement period was Jan 2014-Feb 2015 (13 months), May 2014-Feb 2015 (10 months), and Sept 2014-Feb 2015 (6 months) for Gumbwa, Ndakwera, and Chithumba systems, respectively. Unfortunately, no data could be presented here on Dolo systems as they (at the time of writing) had only recently been installed. Likewise, the data for Chithumba School (comprising two class blocks and a staff room) and a weather station at the same site have not been presented since the systems were vandalized immediately after their installation.

Though the measurements were transmitted to the CMS on hourly increments the RM system captured 15-minute increments. The overall dataset contains a combination of measurement periods: $15 \mathrm{~min}, 30 \mathrm{~min}$, and 1 hour. During the initial data analysis, averages were made for all parameters and calculated values depended on whether 1-hour, 30-minute, or 15-minute increments were desired. Further analysis is envisioned using the more granular data, though for the purposes of this paper 1-hour increments were used. In this case, any subincrements were rounded to the nearest hour and weighted against other measurements at that hour to produce an average hourly value per system in question.

During this process, 262 observations were rejected during data cleaning for "overtime" meaning that no further observation was recorded within the next two hours. These values were treated as outliers. The longest period of down time was 576 hours (24 days) with down times averaging 18 hours.

In terms of RM system resilience to failure, the study observed that the initial installation at Gumbwa site was not much robust as it required frequent maintenance visits. Specifically, it required three visits per year, the most frequent one of all the locations. This particular site was used as a learning curve for the subsequent installations. The improvements made ranged from sensor placement to optimizing software configuration. These changes fostered the resilience of the RM system such that most of the new installations required no maintenance for a period of over two years. 


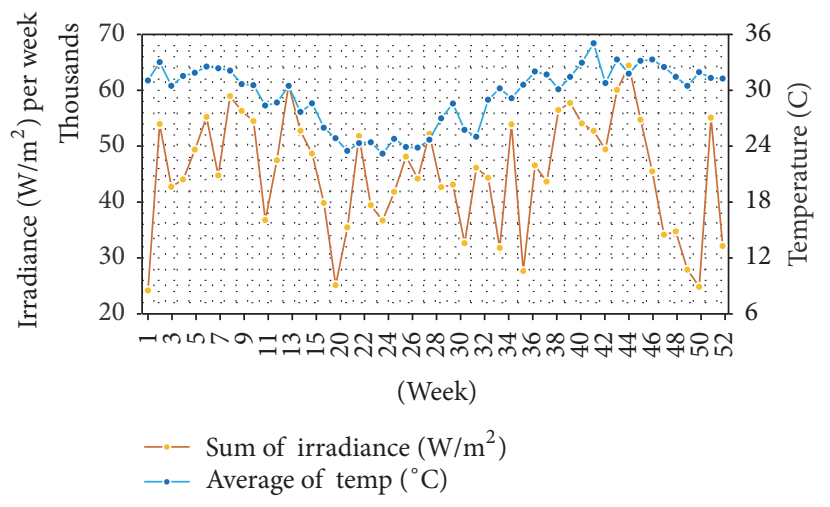

Figure 12: Temperature and irradiance at Gumbwa.

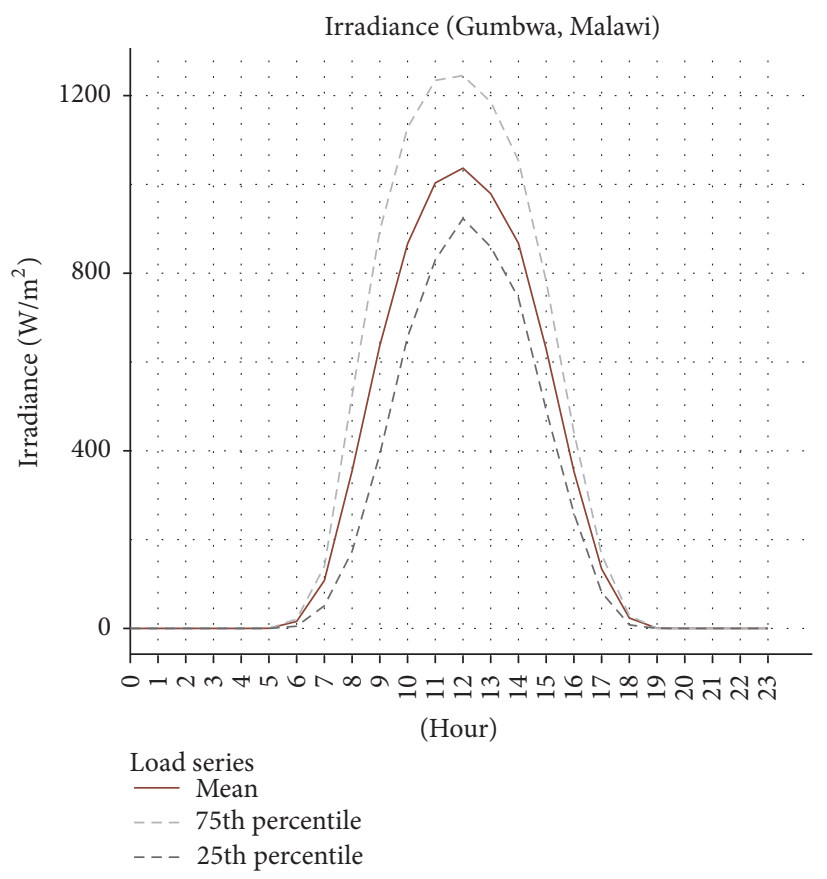

FIgUre 13: Daily irradiance (Gumbwa, Malawi).

The effective operation of the RM system and, hence, its timely reporting of any PV system hiccups had an overall result of enhancing the performance of the PV systems since preemptive maintenance was conducted.

\section{Results}

A selection of the data is plotted to describe the findings on how the system operates. Since data were captured at one point on the hour, it was necessary to make some assumptions in order to calculate consumption and production, for example. However, given the average number of monthly observations made per system, 587, each observation is weighted accordingly.

Figure 12 shows the weekly average temperature and irradiance readings as generated by the weather station located at Gumbwa site. Temperature on the right axis is lowest in around week 24 (early June) at $23^{\circ} \mathrm{C}$ and then peaks to $35^{\circ} \mathrm{C}$ around week 43 (late October). Irradiance is more variable but was generally low around December.

Figure 13 plots the overall hourly irradiance curve over the course of the day. Light picks up readily after 06:00 and peaks at $1,036 \mathrm{~W} / \mathrm{m}^{2}$ at around noon and then goes on to fade by 18:00. Furthermore, the peak hourly irradiance within the highest month (April 2014) was $1,191 \mathrm{~W} / \mathrm{m}^{2}$ and the lowest one (December 2014) was $773 \mathrm{~W} / \mathrm{m}^{2}$. Indeed, most months peaked at nearly $1000 \mathrm{~W} / \mathrm{m}^{2}$ around midday. Daily average irradiance was $7.02 \mathrm{~kW} / \mathrm{m}^{2}$. This is slightly higher than the tilted flat plate estimate of $5.75 \mathrm{~kW} / \mathrm{m}^{2}$ from the OpenEI database [36] but is still reasonable.

Estimating hourly consumption was computed based on point load current and system voltage readings and assumed to be consistent over the previous hour. In some cases, the time between readings had significant gaps (greater than 2 hours). All readings which did not closely follow a preceding reading were removed as it could tend to make the estimate 
TABLE 1: Daily and cumulative system consumption.

\begin{tabular}{|c|c|c|c|c|c|c|c|c|}
\hline \multirow[b]{2}{*}{ Location } & \multirow[b]{2}{*}{ System } & \multicolumn{4}{|c|}{ Daily energy use statistics (Wh) } & \multicolumn{3}{|c|}{ Other usage statistics } \\
\hline & & Min & Mean & Median & $\operatorname{Max}$ & $\begin{array}{c}\text { Cumulative } \\
\text { energy use } \\
(\mathrm{kWh})\end{array}$ & $\begin{array}{l}\text { Dataset range } \\
\text { (days) }\end{array}$ & $\begin{array}{l}\text { Max power } \\
\text { (W) }\end{array}$ \\
\hline \multirow[t]{2}{*}{ Chithumba } & $\begin{array}{l}\text { Maternity } \\
\text { Ward }\end{array}$ & 250 & 773 & 770 & 1533 & 117.45 & 152 & 162 \\
\hline & OPD & 332 & 518 & 489 & 1088 & 62.63 & 121 & 161 \\
\hline \multirow{4}{*}{ Gumbwa } & Classroom 1 & 0 & 85 & 68 & 444 & 28.9 & 339 & 60 \\
\hline & Classroom 2 & 0 & 150 & 102 & 1498 & 50.84 & 339 & 286 \\
\hline & Health post & 7 & 285 & 272 & 541 & 96.71 & 339 & 62 \\
\hline & Staff room & 0 & 97 & 86 & 416 & 33.04 & 339 & 200 \\
\hline \multirow{4}{*}{ Ndakwera } & Classroom 1 & 0 & 180 & 136 & 929 & 48.59 & 270 & 202 \\
\hline & $\begin{array}{l}\text { Maternity } \\
\text { ward }\end{array}$ & 150 & 387 & 370 & 900 & 104 & 269 & 124 \\
\hline & OPD & 0 & 516 & 600 & 1433 & 138.82 & 269 & 225 \\
\hline & $\begin{array}{c}\text { Recovery } \\
\text { ward }\end{array}$ & 0 & 571 & 812 & 1583 & 153.69 & 269 & 386 \\
\hline \multicolumn{6}{|c|}{ Total } & 834.67 & & \\
\hline
\end{tabular}

unreliable. By the end of the monitoring period, $834.67 \mathrm{kWh}$ was consumed by the systems. A more detailed breakdown of the daily and cumulative energy consumption for each system is shown in Table 1. Mean energy use for systems was relatively varied between $85 \mathrm{Wh}$ and $773 \mathrm{Wh}$, which is significant given that many of the systems were similarly sized.

In Figure 14, the average daily load profile for all systems was constructed using averaged hourly sensor readings. The Ndakwera systems clearly had much higher usage, particularly at night. For example, the recovery ward consumed around 65-75 W consistently from 19:00 through 05:00, and the maternity ward used $25-28 \mathrm{~W}$ over the same period. The most plausible explanation to these peak values is that the recovery ward houses guardians and mothers who have delivered and they normally stay for a while (2-3 days) to recuperate. On the other hand, the maternity ward has lower peak values and is mainly used for delivery of babies where mothers and babies are immediately transferred to the recovery ward afterwards. It is interesting to note that the systems had similar designs (equal battery capacity, PV panels, and lighting points). As the systems were being designed, the assumption was that they would have equal loads operating for equal periods. However, that assumption is invalidated as the maternity ward seems to have been oversized.

Of the Gumbwa systems, the health post has the highest nightly load of around $10 \mathrm{~W}$, with the other systems consuming only around 5-6 W. The obvious reason for this is that the health post has several lighting points which are used throughout the night to attend to patients unlike the other systems which are only patronized during the day and late in the evening when learners are available. Gumbwa classroom 2 has a slightly unique profile in that there is a more significant daily load picking up around 09:00-19:00. This is due to the provision of socket outlets for this class. The sockets are used for charging phones and watching TV during the day and late in the evening. Both classroom 2 and staff room have inverters which charge mobile phones for both staff and community.

Gumbwa classroom 2 and Ndakwera classroom 1 have mean values that lie above $75 \%$ for a noticeable period during the day and early evening. This indicates that, the majority of the time, usage is quite lower, though there are some periods where usage is significantly greater than usual, thereby skewing the mean upward.

The weekly load profile for the systems is shown in Figure 15 . Weekend use is not particularly different from normal weekday use for most systems. The Gumbwa staff room typically has a peak during the day between 08:00 and 11:00 of around $11 \mathrm{~W}$ rather than overnight like the other systems. This implies that staff members used the system solely for phone charging and/or laptop use during the day only. There were no activities at night by staff members to utilise the facility. The night load therefore is only for security lights.

By comparing the Gumbwa classrooms and Ndakwera classroom through the weekly load profile, different usage can be observed. Early night usage peaking at around $30 \mathrm{~W}$ during the weekdays at Ndakwera is most likely associated with night study. Classroom 1 at Gumbwa has nightly usage but it peaks at around $7 \mathrm{~W}$ which is more consistent with a security light being used only. Although there is a slight peak for Gumbwa classroom 2 during weekdays, the main night load is around $7 \mathrm{~W}$ with loading primarily over the weekend. Gumbwa classroom 2 has higher usage over the early evening on Friday through Sunday. Peak usage for this system occurs at around 18:00 and is around $38 \mathrm{~W}$ for reasons previously mentioned.

Maintaining battery health is paramount to the overall technical system sustainability. The data captured allowed 

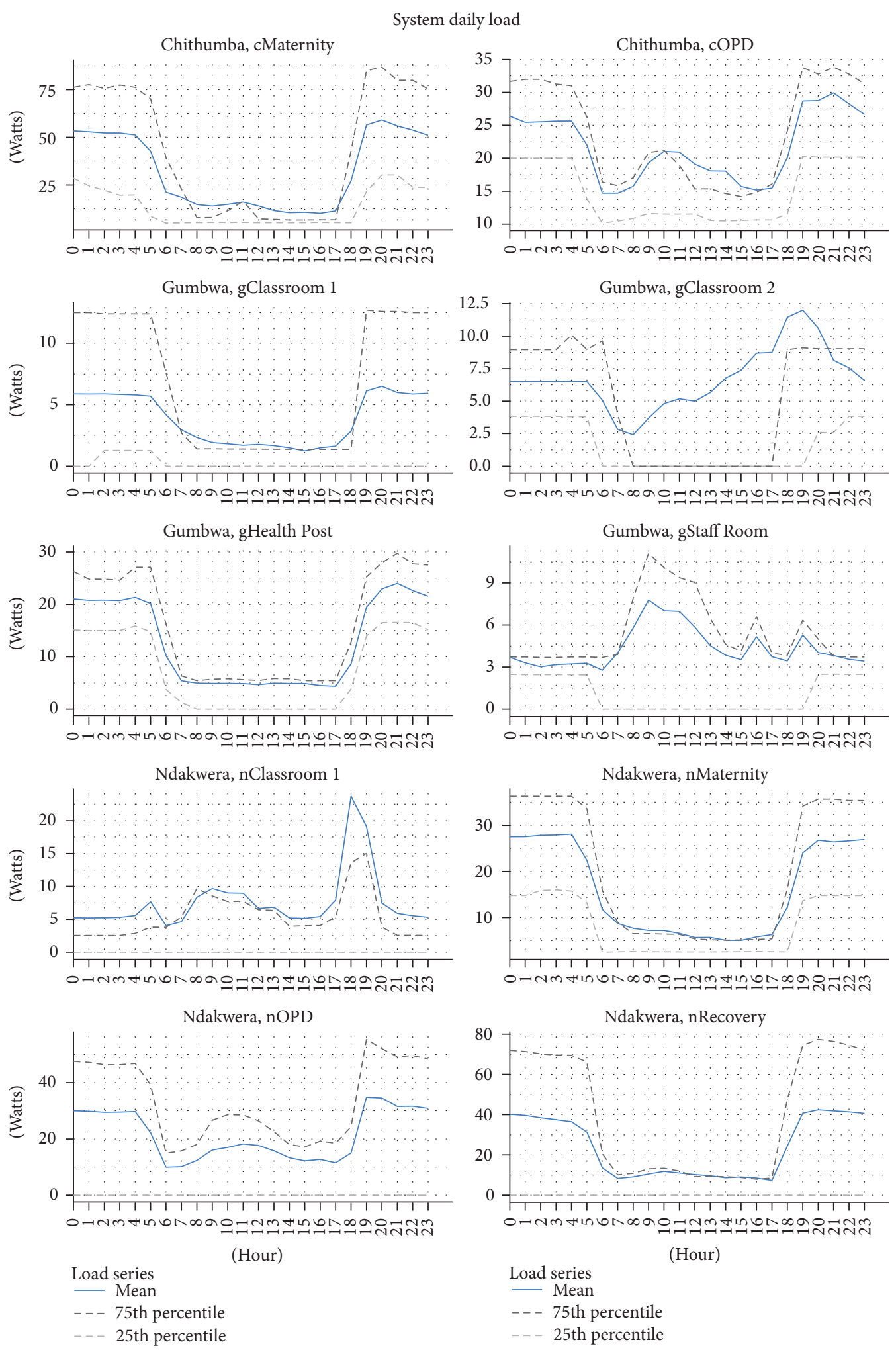

FIgURE 14: Average daily load profile. 


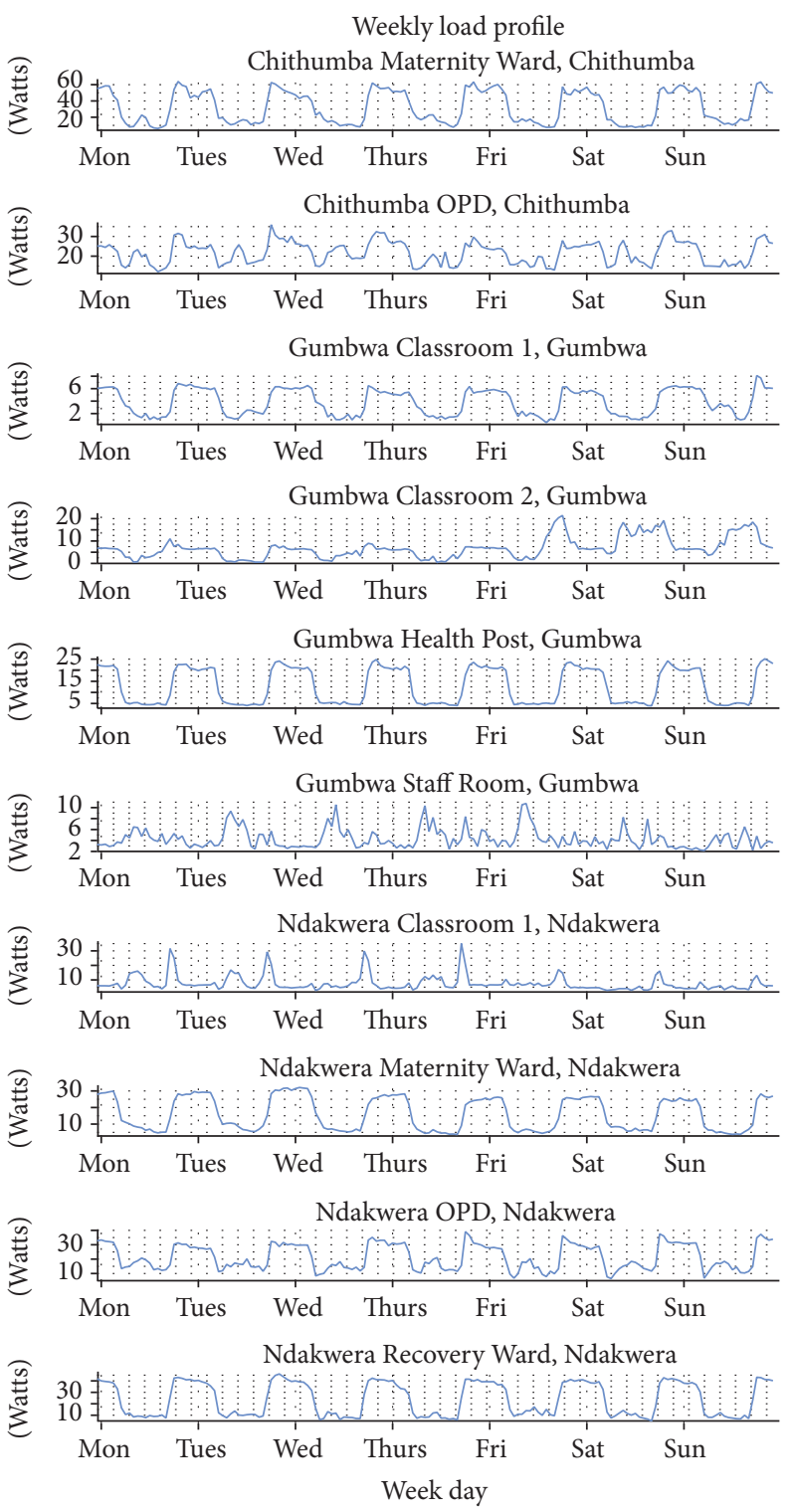

FIGURE 15: Weekly load profile.

for identification of instances where battery voltage was either too low or too high; both situations could lead to damage of the lead acid batteries installed. Figure 16 plots the average, minimum, and maximum battery voltage during each day. The measurement period for a selected group of systems where there were abnormalities in the battery voltages is shown for analysis. The "average" value considers all measured values throughout the day to determine the daily average.

A good rule of thumb for $12 \mathrm{~V}$ nominal lead acid battery systems is that batteries should only ever operate between $11.4 \mathrm{~V}$ and $14.0 \mathrm{~V}$. If functioning properly the charge controller protects the batteries from over- and undervoltage situations by disconnecting the generation source or load, respectively, though the actual operating limits are determined by the settings on the specific charge controller model.
Using this rule of thumb, it becomes apparent that a number of abnormal situations were faced throughout the measurement period. In particular, Gumbwa classroom 1 experienced excessively low voltage in months 3 (Apr 2014), 9 (Oct 2014), and 11 (Dec 2014). In April 2014 the controller developed a fault and was unable to cut the load when the battery level was below the minimum threshold. Figure 17 shows battery voltage profile before and after the controller was replaced. In October 2014 and December 2014, on the other hand, it was noted that the CB was tripping off typically between 09:00 and 12:00. Through inquiry, it was noted that learners had been doing such a malpractice. As stated previously, the recording of a zero voltage as a result of the tripping of the $\mathrm{CB}$ was a design flaw of the RM system and was rectified eventually. The Gumbwa staff room had low voltage in month 1 (Feb 2014). This occurred due to a technical fault 

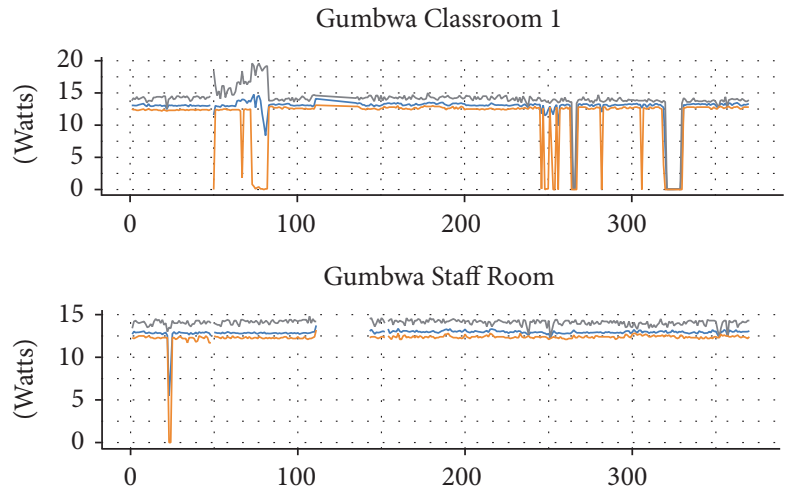

Ndakwera Maternity Ward

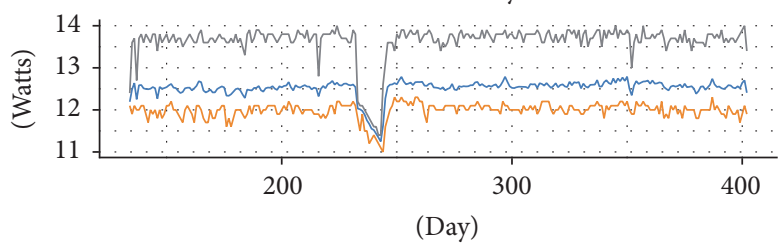

Battery voltage

- Average $V /$ day

- Max $V /$ day

- Max $V /$ day

FIGURE 16: Battery voltage on selected systems.

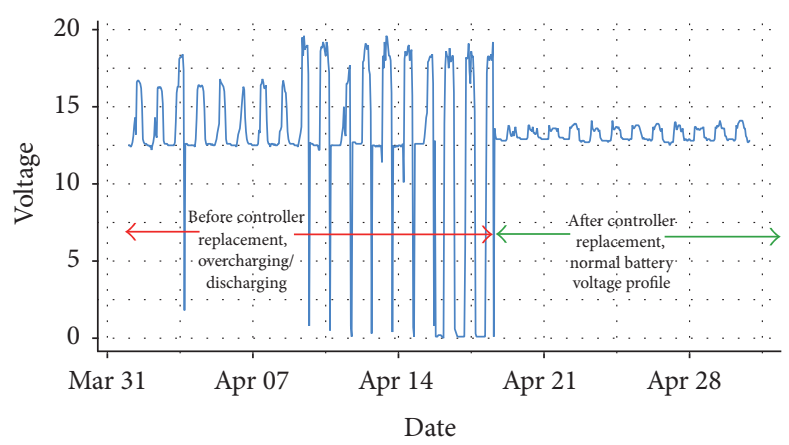

FIgURE 17: Battery voltage profile for Gumbwa Class Room 1.

which resulted in short circuiting of the battery bank. At the Ndakwera health centre, the system had a prolonged period between months 8 (Sept 2014) and 9 (Oct 2014) where the battery voltage dipped. Investigations revealed that the PV panel CB was accidentally tripped off; hence, batteries were not able to charge during the day until the problem was rectified.

With near real time monitoring, an operator can be alerted to the abnormal situation and respond accordingly. In a conventional system without RM, the community operators may not notice the problem until much later. There is a potential risk of misdiagnosis if they try to troubleshoot the problem without sufficiently trained personnel. With the available data, clues to the potential problems can be identified much more quickly and accurately. Low voltage, beyond the threshold set by the charge controller, for example, should only occur if the charge controller's low voltage disconnection has failed or if the user has somehow bypassed the controller. Each system is designed based on an expected load demand and resource (solar in the case of these systems). Although approaches differ, most off-grid systems cannot sustain full peak loading for an extended period. System design estimates often assume a profile such as that shown in Figure 14. Therefore, if additional unplanned load is consistently appearing on the system, the ability of the system to supply all loads may be tested.

Gumbwa project demonstrates how sustainability can be improved through early identification of potentially damaging user behaviour. An example would be connecting a large amplifier to listen to music on the weekend or after hours, a situation which was experienced on 15 February 2014 on a Gumbwa classroom 2 system as shown in 


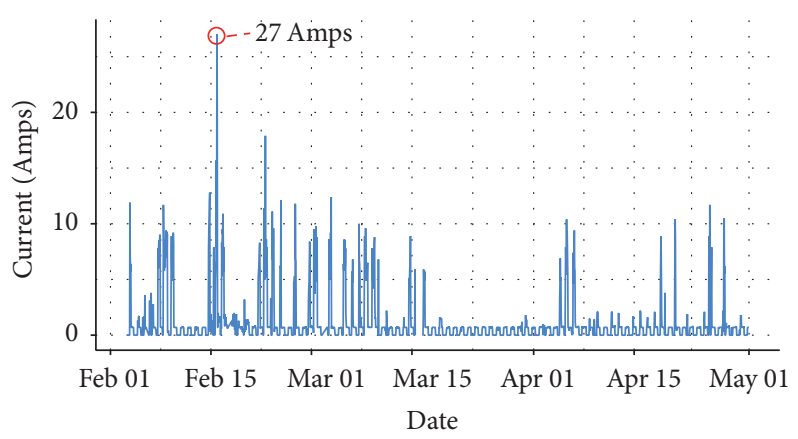

FIgure 18: Gumbwa Class Room 2 load current profile.

Figure 18. Here, current rose to an abnormal value of about 27 Amps - triggering a fault alarm in the process. In the case of the Gumbwa project, which supplies lighting for 1037 primary school pupils and 11 teachers, this will impact the benefit they actually receive which may be the desired community benefit. With RM, the community can be notified, within the hour, to stop or reduce the undesirable system usage.

\section{Discussion of Results}

Whereas the preceding sections have established the "where," "what," and "how" of the deployment of RM, this section seeks to build the argument for the value of RM and its impact on sustainability and development outcomes of the associated projects, discuss challenges that were faced, and finally consider the future technology development and potential scale-up/out.

\subsection{Value of RM for Institutional Solar PV Community Energy} Projects on Impacts and Sustainability. The RM system provides an accurate and detailed data on key impact data for a community energy project. This comes primarily in the form of an energy usage; kilowatt hours used is probably the most convenient measure. An acceptable voltage reading and a reasonable charging and usage pattern, as was shown, is a good indicator that a system is functioning at all. Basic data analysis of load patterns was demonstrated to be useful in showing exactly how the system is being used. Comparison of the RM-enabled classrooms showed quite different usage patterns especially when compared over daily and weekly segments. Where a development outcome is desired, tracking nightly usage for studying or availability of security lighting as the case may be can be relatively easily determined without resorting to manual methods of logbooks. Indeed, a more complex RM design could include separate sensors for different circuits such as lighting, mobile phone charging, and vaccine refrigerators. Impact data such as power usage can then be incorporated into project monitoring and evaluation systems with relative ease.

From a technical sustainability standpoint, a major benefit of the RM system was the capability to identify system "fault" situations. This was the combination of a near realtime display of the system parameters, a function to check whether these parameters were within acceptable boundaries, and a functionality to reach out to both the users and operators via SMS. Indeed, this occurred at the systems on more than one occasion, even within the first year of the operation. In the case of Gumbwa classroom 2, two issues were at play: a malfunctioning charge controller and a large unexpected load. Without RM this type of system usage can be very difficult to spot, especially if usage of the system is not tightly controlled by the community. Furthermore, users may not be fully aware of the limited supply that they have nor the energy use of the devices they attach to the system. Without an exceptionally technically astute local operator, the situation could potentially persist until an infrequent periodic visit by a technical support person or until the system was damaged and required replacement.

With improved ability to identify fault situations, there is an implication on how maintenance of systems is handled. Community energy projects in Malawi are, generally speaking, located in very rural areas. The MREAP project as a whole was comprised of projects that were in the most remote locations [37]. In districts where MREAP was present, only $3.2 \%$ of households used electricity as a main source of lighting compared to $7.6 \%$ at the national level [38]. The provision of sufficient maintenance is a major challenge be it market based or otherwise for two reasons: availability of reliable information and cost. The RM deployment in MREAP has shown that very specific, valuable information can be made quickly available to both operator and user. Without this, an operator will have to rely on vague and likely unreliable fault identification from the user, who may not be qualified to determine this reporting or would need to visit the project to assess its condition. Under the assumption that technicians who can maintain off-grid solar PV systems are not located in the remote villages themselves, each field maintenance visit represents a substantial transaction cost. RM therefore would allow for a maintenance strategy that requires maintenance trips only when needed and has a reliable data for the contractor beforehand, which could be more economically optimal.

In addition to use of RM during operations, the data generated represents an important base for project design for future similar community energy projects. Though the dearth of information on community energy projects is being improved upon, partly through the efforts of MREAP as a whole [17, 39], little detailed information on usage 
patterns for these systems is widely available. Technical needs assessments including an estimation of expected load by type and quality conducted prior to project installation are required to forecast expected usage for the project. This information is typically used as an input into system sizing calculations. As was shown in systems at Ndakwera health centre, there is an error in this process. By building robust datasets of load patterns, the technical needs assessment can be strongly supported by empirical evidence thereby reducing the error in the design. Furthermore, availability of data, both ex post and in real-time, can be a boon for research in improving long-term sustainability and deployment of effective business models for the context.

\subsection{Challenges Faced}

5.2.1. Operational Challenges. The main challenge experienced was unreliability of the mobile network. As discussed previously, the system aimed at collecting the performance metrics from the remote PV system. This data was captured through SMSs which were sent through the GSM network operated by Telekom Networks Malawi (TNM) Limited. The network, thus, was a major dependency for successful operation of the RM. Some rural areas are not fully covered by mobile networks, so its absence would undermine the viability of RM. Specifically, Ndakwera has no access to TNM mobile network used in the CMS and this required the use of another available GSM network operated by Airtel Malawi Limited. Worse still, Airtel network is very unreliable at this site and resulted in loss of several SMSs. It would be interesting to investigate the use of other communication and routing protocols to circumvent this challenge. For instance, for routing protocols, an efficient state-based hybrid protocol for mobile nodes would be opted for as suggested in [40]. For communication protocols, the RM devices used in this study had an option to use Wi-Fi. However, since Wi-Fi propagates well using LOS communication mode, it would require setting up repeaters on nearby hills/mountains to propagate the signal all the way to the CMS. Unfortunately, the obvious implication of this is a substantial increase in the capital cost of the RM system.

There was also a challenge in the operation of RM culminating from installation flaws especially for some nodes at Gumbwa site. In these nodes, it was observed that Circuit Breakers (CBs) were installed between Voltage Sensors and battery banks on the lines coming from charge controllers. This means that when the $\mathrm{CB}$ was tripped intentionally or otherwise, the battery Voltage Sensor read a zero voltage which was reported as a full battery discharge resulting in the triggering of a fault alarm. It was discovered that pupils were playing around with the CBs and tripping them in the process. When that flaw was noted, it required redesigning of the RM by placing the Voltage Sensors after the CB. It may be appropriate in future deployments to consider monitoring user malpractice especially in classrooms where pupils may pose threats to the operation of the PV system. Furthermore, user training should incorporate this lesson to try to prevent the behaviour.
Additionally, there was difficulty in achieving LOS communication mode in most cases. This is because most of the RS units were installed indoors where the PV control box was situated. Although most of the nodes were within the possible LOS communication range of $100 \mathrm{~m}$, absence of LOS thus resulted in loss of communication between the substation and the main station and hence loss of data. This prompted the implementation of several main stations per site. Future deployments should consider installing the communication units on roof-tops rather than indoors. However, one needs to first explore the implication of using longer data cables from sensors installed close to the PV control box to the data processing unit at the roof-top. The use of directional antennas and/or other communication protocols (for instance, radio frequency) may also be an area of further improvements to this challenge.

5.2.2. PV System Vandalism Reporting Challenge. Although the vandalism of the PV system did not directly impact the operation of the RM system, it was noted that the reporting of such malpractices was not adequately addressed by the RM system. For instance, there were cases at Ndakwera and Chithumba where one or more batteries were illegally removed from the battery bank cage without the RM capturing such. Incorporation of a mechanism to detect such vandalism would allow local operators of the PV facility to intervene as quickly as possible.

\subsection{Readiness for Scaling}

5.3.1. Availability of Components. Although most of the components and devices employed in this study were sources from overseas, it is still expected that their availability is guaranteed and that they cannot hinder scaling of the technology. This paper demonstrated a promising small scale pilot, but a larger deployment with a longer monitoring time frame is needed to fully validate equipment performance. Additionally, procurement associated with importing any foreign product will limit speed of deployment. A local option or more developed supply chain for the components used here would alleviate this issue.

5.3.2. Restrictions and Dependencies. As previously discussed, the RM system (particularly the RS) becomes more economically attractive with an increase in the number of independent PV nodes linked to one main station. Although [41] argues that there is no restriction on the number of substations (children nodes) to be able to connect to one main station (coordinator node) in the ZigBee platform, especially when routers are used, the Waspmote data processing board may have a limit on the number of these children nodes. With a volatile memory of $8 \mathrm{kB}$ for the current ATmega 1281 Microcontroller Unit [33], it may be impossible to successfully embed a fully functional code in the Microprocessor Unit (MPU) as the number of children nodes increases. Furthermore, [42] outlines other challenges associated with an increased number of children nodes to be managed by one coordinator node. These include, but not limited to, network instability and data packet losses. With 
the current deployment, it is estimated that a maximum of eight children nodes can effectively be connected to the main station to permit all the design requirements of capturing and analysing data and sending fault alarms accordingly. As such, when the number of independent PV nodes at one facility increases beyond this limit, it is necessary to deploy more than one main station to overcome the aforementioned challenges.

Even if it was possible to integrate many substations to one main station, it would be challenging to circumvent the 160-character limitation for the SMS transmission. As more and more substations are integrated, the need to concatenate the data into a single SMS string may become a limit. Therefore, a large scale rollout may require that the data be broken into several sections and sent one by one within a given period of time. The economic risk of the running costs of the RM system could be painfully high. The current deployment integrated a maximum of four substations to a single main station and one SMS was sent every two hours containing every hour's data. This implies that it was possible to integrate eight substations by increasing the SMS sending rate from every two hours to every hour. Nevertheless, if the number of monitored parameters could be reduced, more substations could then be connected without increasing the SMS sending rate and still operate within the 160-character limit. The downside of this balancing act is that fewer data points may limit potential monitoring capability.

Finally, there is really no limitation of the operational capabilities of the Central Monitoring Station (CMS). In fact, the total RM cost per monitored site reduces with an increase in the number of the sites managed by one CMS. It is therefore envisaged that, with well-designed software, the CMS would be very suitable for a large scale rollout of the RM-enabled PV systems. However, it would be interesting to investigate how the system can interact with the maintenance process in terms of personnel and ability to intervene.

5.3.3. Cost Implications. Under the MREAP needs assessment, 20 rural public facilities were identified and targeted for intervention in just Chikwawa district alone. However, there are many more public facilities nationwide which lack access to electricity and could potentially be addressed by an off-grid solution. According to UNESCO, only $10 \%$ of primary schools and $53 \%$ of secondary schools had access to electricity in 2012 [43]. Statistics on access to electricity at health facilities in Malawi is unavailable, though anecdotally it is thought to be similarly low.

Economic costs for a national rollout of solar PV with RM monitoring at all rural public facilities would represent a significant national investment. Using capital and operating expense profile for the systems deployed is not appropriate given the likely scaling advantages, resulting in lower incremental costs and socioeconomic benefits that national deployment would entail. It is expected that a national rollout of off-grid solar PV with RM would be trading off a higher cost versus improved reliability and sustainability of a nonRM rollout. A study comparing the cost and benefits of this choice is beyond the scope of this paper but of serious academic interest.

\subsection{Extensions for RM and Implications for Future Research}

5.4.1. Local Use of RM. The deployment model in WASHTED's SEP was based on a strong community ownership and management model. In each community, an energy committee was setup to provide oversight over the project and make decisions on behalf of the project. After installation of the equipment WASHTED provided limited technical maintenance support, when required. The RM system therefore provided data-driven input to the team in Blantyre on when and what type of maintenance intervention was required. However, for communities, the interaction with RM was, for the most part, minimal. The exception was when alarms would be automatically generated by the software prompting a warning SMS to be sent to both the operator (WASHTED) and the community. Real-time availability of data or analysis was not available to the community, though it could be argued that capacity to interpret results would be limited. Extending this functionality to allow for better understanding of the system, particularly in the area of types of usage and capability of the system to handle usage patterns, would be empowering to the community responsible for managing the project. Granting that community projects have a wide range of ownership and operational structures, models which seek to empower local management and control may be able to utilise RM data fully.

Finally, the degree to which RM technology can be used to support potential micro-utilities and the off-grid supply chain as a whole is unknown. The relevant local key case study is the MEGA model [44], but this is by no means the only option available ([45] describes the case of the rural ESCOs in Zambia as one potential model); this paper can speculate potential options including rural operators covering a service area, solar PV contractors managing maintenance contracts with community energy projects, or district officers overseeing rural systems. To clarify this, the paper is arguing that RM can be a support tool by providing a wealth of information that was previously unattainable for a variety of organization modes (and markets), not that this study necessarily supports any particular arrangement ([46] theorizes the uses and potential economic gains for a RM deployment in Ethiopia using the SIMbaLink technology, which may be relevant for discussions around potential benefits to particular arrangements.).

5.4.2. RM System and Implementation Costs. Despite its benefits, use of RM is not an absolute requirement for community energy projects. Its integration has an implication on the economics of the project itself. The RM deployed in MREAP has both initial capital and ongoing operating costs, which must be accounted for by project implementers and communities that go on to manage the project. For the four projects deployed in Chikwawa, RM equipment represented around $25 \%$ of total capital costs per location. Viability of RM for this market would need to be accompanied by a positive net impact on the project-level economics. Further (pecuniary) evidence is needed to verify the returns to implementation of RM. The data in this paper suggest that increased reliability and better utilisation of any revenue 
generating activities associated with the project's energy use may be a key mechanism for any returns due to RM. Since offgrid community energy projects in Malawi have historically struggled with sustainability, every opportunity to improve the equation is important to exploit.

5.4.3. Use of Data to Support Decision Making. With greater availability of data from RM systems there is an opportunity to develop decision making support and control tools for the potential users. In the context of off-grid community energy projects in Malawi, tools to support better sustainability outcomes would be most desirable. This would likely require integration of models and forecasting techniques so that sustainability factors and current performance against them were available and interpretable by users. Within MREAP's RM deployment, the feedback loop between current usage and forecasted future sustainability was limited; operators in WASHTED made some judgments on current system sustainability followed by corrective actions; however, no systematic method was used.

Improved data available on usage can be the foundation for more advanced project and programme design. In addition to forecasting sustainability, data on current and near future system capacity could be used by project owners to better optimize usage of the system. An approach could be layering RM data, metering, with a pricing mechanism that is aware of current capability of the system to meet demand and act by providing the right signals to customers.

5.4.4. Control Tools. Control tools which can take data from $\mathrm{RM}$ and issue automated or direct remote control actions are further advancements on the technology. Controls within the PV systems deployed were manual and unlinked to the RM system. The operator is therefore in many ways an observer to the system and only able to correct a situation through direct intervention or by offering guidance to the local community. This can be costly and risky. In advanced power systems, control equipment is a main feature that is used to protect the system and ensure high levels of reliability. These needs are no different for the relatively basic off-grid solar PV systems often deployed in Malawi. Successful integration of control technology could, either automatically or by local and/or remote operator instruction, limit some uses that are undesirable such as overuse of circuit tied to a television. When combined with sustainability models, it could reduce allowed use before a forecasted low production period that occurs periodically with renewable energy sources or enforce market based limitations. Although the systems monitored in MREAP were all stand-alone solar PV installations, implementation of RM in more advanced systems such as mini-grids may be a further justification for more control capability.

\section{Conclusions}

This research presented results from a novel deployment of a unique RM system utilising WSN in rural off-grid solar PV systems in Malawi. The technology successfully captured detailed operational data which validated the WSN configuration. Operational data including day and weekly load profiles, energy use, weather parameters, and fault situations were analysed. The data produced is a step change improvement for operational analysis and can be used in wider sustainability studies, which are future areas of research. Using the results, it has been argued that inclusion of RM systems could improve individual solar PV installations at rural public facilities. The small scale pilot incurred significant costs to include the RM and a larger rollout would require a more extensive cost benefit analysis.

\section{Conflicts of Interest}

The authors of this manuscript declare that the devices and software applications used in this research were sorely selected on a professional basis. There is no direct financial relation whatsoever with the trademarks mentioned in this manuscript that might lead to conflicts of interest.

\section{Acknowledgments}

This research was funded by the Scottish Government under the Malawi Renewable Energy Acceleration Programme (MREAP). Furthermore, the authors would like to acknowledge the input from colleagues at WASHTED and University of Strathclyde and the communities of Ndakwera, Gumbwa, Dolo, and Chithumba for their cooperation.

\section{References}

[1] UNSD, United Nations 2015: Time for Global Action, United Nations Sustainable Development, 2015, http://www.un.org/ sustainabledevelopment.

[2] IEA, "World Energy Outlook," January 2015, http://www.iea .org/publications/freepublications/publication/WEB_WorldEnergyOutlook2015ExecutiveSummaryEnglishFinal .pdf.

[3] IEA, World Energy Outlook, 2014, http://www.iea.org/publications/freepublications/publication/WEO2014_free.pdf.

[4] J. Terrapon-Pfaff, C. Dienst, J. König, and W. Ortiz, "A crosssectional review: impacts and sustainability of small-scale renewable energy projects in developing countries," Renewable and Sustainable Energy Reviews, vol. 40, pp. 1-10, 2014.

[5] IEG, The Welfare Impact of Rural Electrification: A Reassessment of the Costs and Benefits, Independent Evaluation Group (IEG): World Bank, Washington, DC, USA, 2008.

[6] M. Kezunovic and A. Bose, "The future EMS design requirements," in Proceedings of the 46th Annual Hawaii International Conference on System Sciences (HICSS '13), Washington, DC, USA, January 2013.

[7] M. P. McHenry and D. Doepel, “The 'low power' revolution: rural off-grid consumer technologies and portable micropower systems in non-industrialised regions," Renewable Energy, vol. 78, pp. 679-684, 2015.

[8] D. F. Frame, P. M. Dauenhauer, K. Tembo, C. Currie, and G. W. Ault, "An integrated approach to energy for development: case study of the malawi renewable energy acceleration programme," in Proceedings of the 3rd IEEE Global Humanitarian Technology Conference (GHTC '13), pp. 72-77, IEEE, San Jose, Calif, USA, October 2013. 
[9] University of Strathclyde, "Energy in Malawi," March 2015, http://www.strath.ac.uk/engineering/electronicelectricalengineering/ourinternationalprogrammesprojects/malawirenewableenergyaccelerationprogramme/.

[10] G. Davis, R. MacKay, M. MacRae et al., "Supporting Community Energy Development in Malawi: A scoping study for the Scottish Government," August 2011, http://www.gov.scot/ Resource/Doc/107396/0122148.docx.

[11] K. Tembo, M. Mafuta, and P. Dauenhauer, Solar PV Strategic Energy Project in Southern Malawi: Development, Design, and Impact, Malawi Renewable Energy Acceleration Programme (MREAP), Blantyre, Malawi, 2015.

[12] S. J. Masangwi, Methodology for Solar PV Needs Assessment in Chikwawa, Southern Malawi, University of Strathclyde, Glasgow, UK, 2015.

[13] H. Louie, P. Dauenhauer, M. Wilson, A. Zomers, and J. Mutale, "Eternal light: ingredients for sustainable off-grid energy development," IEEE Power and Energy Magazine, vol. 12, no. 4, pp. 70-78, 2014.

[14] D. Frame, K. Tembo, M. J. Dolan, S. M. Strachan, and G. W. Ault, "A community based approach for sustainable off-grid PV systems in developing countries," in Proceedings of the IEEE PES General Meeting: The Electrification of Transportation and the Grid of the Future, Detroit, Mich, USA, July 2011.

[15] C. W. Lee and J. Zhong, “Top down strategy for renewable energy investment: conceptual framework and implementation," Renewable Energy, vol. 68, pp. 761-773, 2014.

[16] E. Ilskog and B. Kjellström, "And then they lived sustainably ever after?-Assessment of rural electrification cases by means of indicators," Energy Policy, vol. 36, no. 7, pp. 2674-2684, 2008.

[17] S. Feron, "Sustainability of off-grid photovoltaic systems for rural electrification in developing countries: a review," Sustainability, vol. 8, no. 12, pp. 1326-1352, 2016.

[18] C. Zalengera, R. E. Blanchard, P. C. Eames, A. M. Juma, M. L. Chitawo, and K. T. Gondwe, "Overview of the Malawi energy situation and A PESTLE analysis for sustainable development of renewable energy," Renewable and Sustainable Energy Reviews, vol. 38, pp. 335-347, 2014.

[19] C. Currie, G. Banda, H. Dinsmore, S. Henderson, and S. O. Reilly, Evaluation of Off-Grid Community Managed Renewable Energy Projects in Malawi, University of Strathclyde, 2012.

[20] F. D. J. Nieuwenhout, A. Van Dijk, P. E. Lasschuit et al., "Experience with solar home systems in developing countries: a review," Progress in Photovoltaics: Research and Applications, vol. 9, no. 6, pp. 455-474, 2001.

[21] J. E. Elusakin, O. O. Ajide, and J. C. Diji, "Challenges of sustaining off-grid power generation in Nigeria rural communities," African Journal of Engineering Research, vol. 2, no. 2, pp. 51-57, 2014.

[22] D. Palit, "Solar energy programs for rural electrification: experiences and lessons from South Asia," Energy for Sustainable Development, vol. 17, no. 3, pp. 270-279, 2013.

[23] P. Dauenhauer and D. Frame, "Sustainability analysis off-grid community solar PV projects in Malawi," in Proceedings of the IEEE Global Humanitarian Technology Conference (GHTC '16), October 2016.

[24] M. Mafuta, M. Zennaro, A. Bagula, G. Ault, H. Gombachika, and T. Chadza, "Successful deployment of a wireless sensor network for precision agriculture in Malawi," International Journal of Distributed Sensor Networks, vol. 9, no. 5, 2013.
[25] S. M. Abd El-Kader and B. M. Mohammad El-Basioni, "Precision farming solution in Egypt using the wireless sensor network technology," Egyptian Informatics Journal, vol. 14, no. 3, pp. 221-233, 2013.

[26] R. Kamath, M. Balachandra, S. Prabhu, and A. Akhauri, "Precision agriculture in India using wireless sensor," in Proceedings of the 3rd International Conference on Computational Methods in Engineering and Health Sciences (ICCMEH '16), Kitakyushu, Japan, December 2016.

[27] S. Ali, F. Khan, and U. Taj, "Forest fire monitoring using wireless sensor networks-a survey," International Journal of Engineering Science and Computing (IJESC), vol. 6, no. 6, pp. 70247027, 2016.

[28] A. Molina-Pico, D. Cuesta-Frau, A. Araujo, J. Alejandre, and A. Rozas, "Forest monitoring and wildland early fire detection by a hierarchical wireless sensor network," Journal of Sensors, vol. 2016, Article ID 8325845, 8 pages, 2016.

[29] J. Fernández-Berni, R. Carmona-Galán, J. F. Martínez-Carmona, and Á. Rodríguez-Vázquez, "Early forest fire detection by vision-enabled wireless sensor networks," International Journal of Wildland Fire, vol. 21, no. 8, pp. 938-949, 2012.

[30] A. Parikh, F. Pathan, B. Rathod, and S. Shah, "Solar panel condition monitoring system based on wireless sensor network," International Journal of Science, Engineering and Technology Research (IJSETR), vol. 4, no. 12, pp. 4320-4324, 2015.

[31] K.-H. Chao, P.-Y. Chen, M.-H. Wang, and C.-T. Chen, "An intelligent fault detection method of a photovoltaic module array using wireless sensor networks," International Journal of Distributed Sensor Networks, vol. 10, no. 5, 2014.

[32] M. J. Prieto, A. M. Pernía, F. Nuño, J. Díaz, and P. J. Villegas, "Development of a wireless sensor network for individual monitoring of panels in a photovoltaic plant," Sensors, vol. 14, no. 2, pp. 2379-2396, 2014.

[33] Libelium, Waspmote Datasheet, vers 4.4, Libelium Comunicaciones Distribuidas S.L, Zaragoza, Spain, 2013.

[34] A. Salleh, M. K. Ismai, N. R. Mohamad, M. Z. A. Abd Aziz, M. A. Othman, and M. H. Misran, "Development of greenhouse monitoring using wireless sensor network through ZigBee technology," International Journal of Engineering Science Invention, vol. 2, no. 7, pp. 6-12, 2013.

[35] Phidgets, "Voltage/Current," Phidgets Inc, January 2015, http:// www.phidgets.com/products.php? category $=8$.

[36] UNEP, "Solar and Wind Energy Resource Assessment-Datasets," 2015, http://en.openei.org/wiki/SWERA.

[37] C. Currie, G. Davies, and E. Young, Process Evaluation of Community Energy Development Programme Projects, University of Strathclyde, Glasgow, UK, 2015.

[38] NSO, “Third Integrated Household Survey (IHS3) 2010/2011," 2012, http://www.nsomalawi.mw/images/stories/data_on_line/ economics/ihs/IHS3/IHS3_Report.pdf.

[39] C. Currie, G. Banda, H. Dinsmore, S. Henderson, and S. O. Reilly, Evaluation of Off-grid Community Managed Renewable Energy Projects in Malawi, University of Strathclyde, Glasgow, UK, 2012.

[40] M. M. Umar, N. Alrajeh, and A. Mehmood, "SALMA: an efficient state-based hybrid routing protocol for mobile nodes in wireless sensor networks," International Journal of Distributed Sensor Networks, vol. 12, no. 2, pp. 1-11, 2016.

[41] Libelium, Waspmote Programming Guide, vers 4.1, Libelium Comunicaciones Distribuidas S.L, Zaragoza, Spain, 2013. 
[42] A. Shaikh and S. Pathan, "Research on wireless sensor network technology," International Journal of Information and Education Technology, vol. 2, no. 5, pp. 476-479, 2012.

[43] UIS, "Education MetaData: access to basic services in public schools by level of education," March 2015, http://data.uis.unesco.org/index.aspx?queryid=194.

[44] BIF, "MEGA-A commercial approach to off-grid power in rural Malawi," December 2013, http://www.inclusivebusinesshub .org/wp-content/uploads/2016/05/Deepdive_MEGA_HUB-10 .pdf.

[45] X. Lemaire, "Fee-for-service companies for rural electrification with photovoltaic systems: the case of Zambia," Energy for Sustainable Development, vol. 13, no. 1, pp. 18-23, 2009.

[46] N. Schelling, M. J. Hasson, S. L. Huong et al., "SIMbaLink: towards a sustainable and feasible solar rural electrification system," in Proceedings of the 4th ACM/IEEE International Conference on Information and Communication Technologies and Development (ICTD '10), London, United Kingdom, December 2010. 

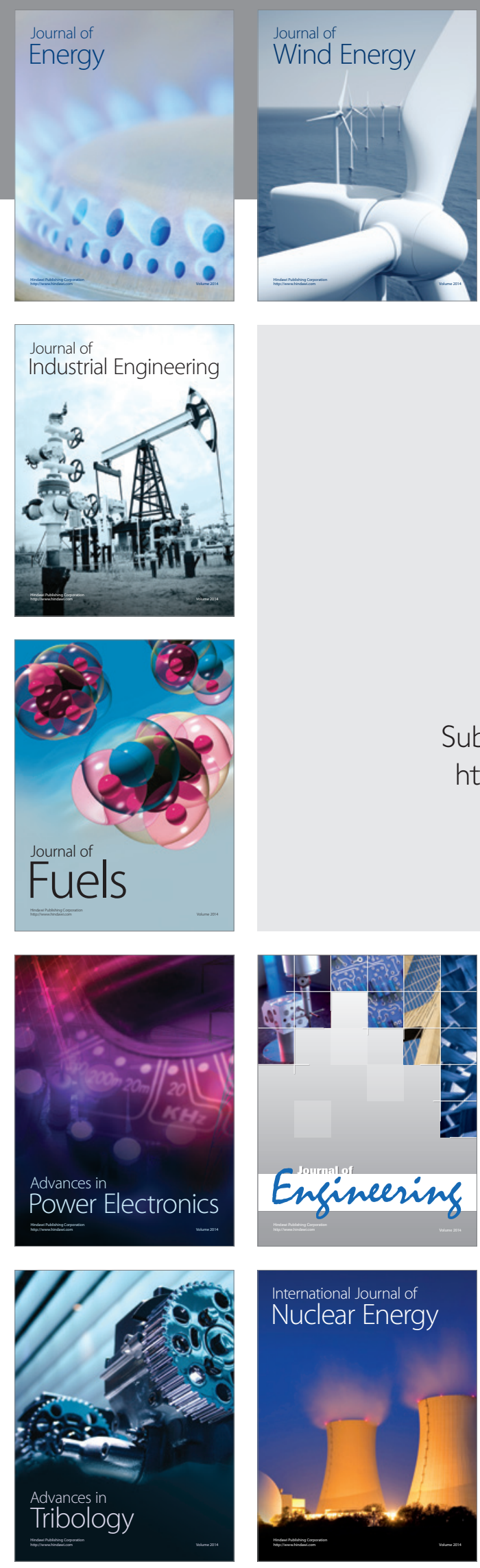

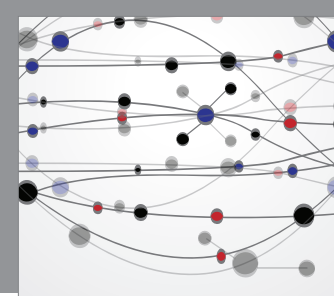

The Scientific World Journal
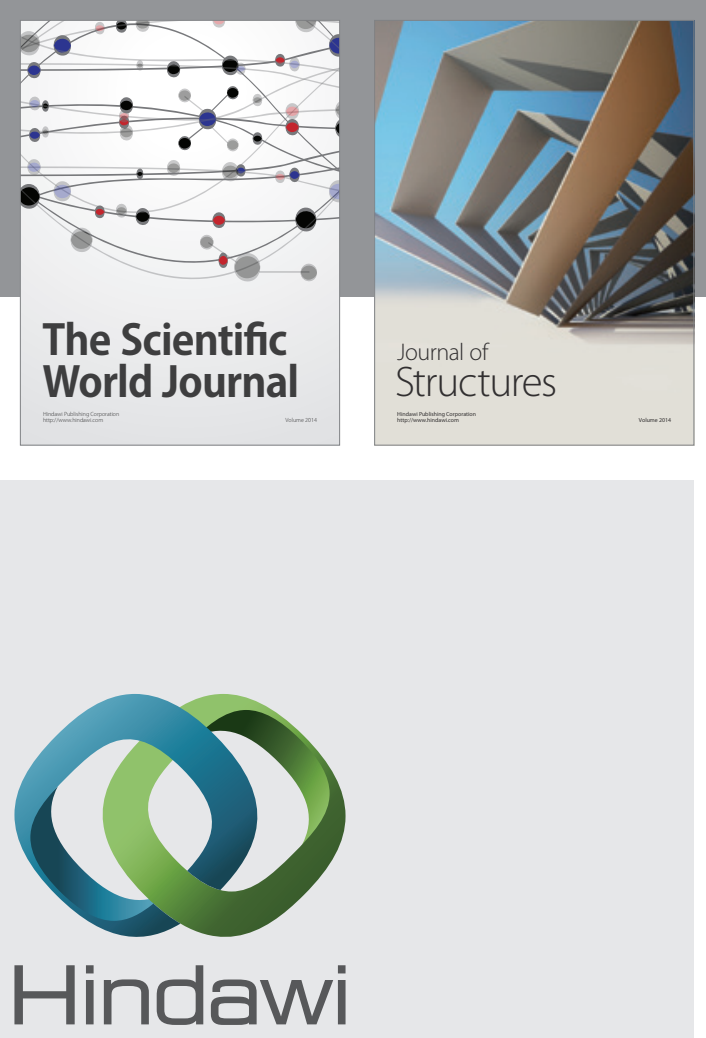

Submit your manuscripts at

https://www.hindawi.com
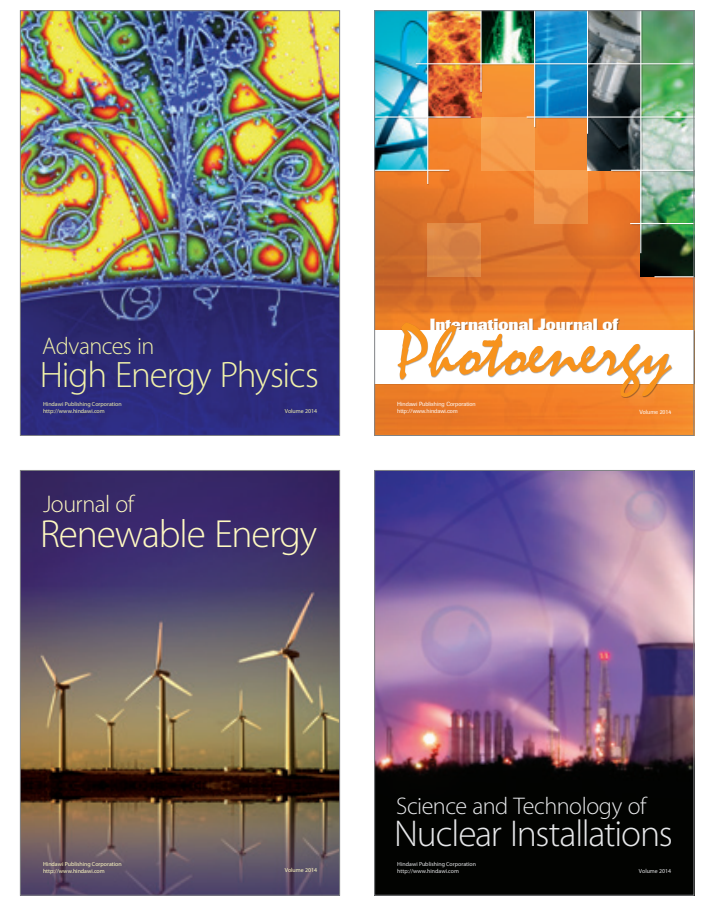
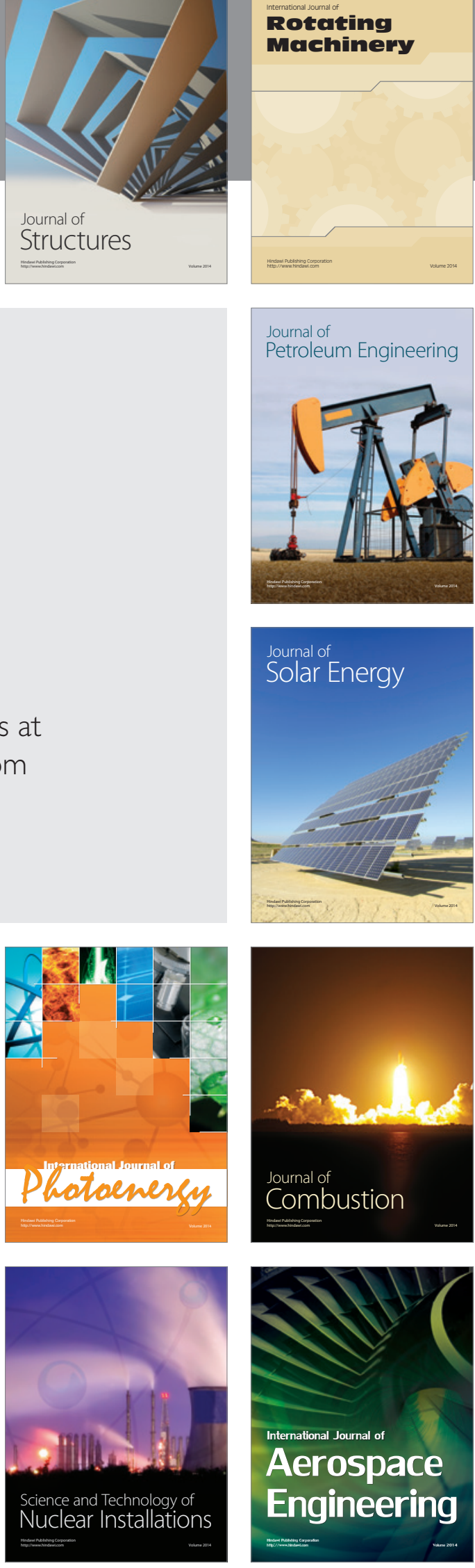